\title{
Perspektive und empathische Resonanz: Vergegenwärtigung anderer Sichtweisen
}

\author{
SUSANNE SCHMETKAMP
}

\section{EINLEITUNG}

Richard Rorty vertritt in seinem Aufsatz »Der Roman als Mittel zur Erlösung aus der Selbstbezogenheit« die These, dass fiktionale Werke wie etwa Romane uns die Möglichkeit böten, uns aus der eigenen egoistischen Perspektive zu lösen und so unseren Horizont ethisch wertvoll zu erweitern: »Um sich zu verändern, kommt es darauf an, an einen Ort gebracht zu werden, von dem aus Neues sichtbar wird «. ${ }^{1}$ Diese Art der Selbsttransformation setze voraus, dass wir unseren Standort änderten. Mit anderen Worten: Dass wir unsere Perspektive verändern, um die Perspektiven anderer zu verstehen. ${ }^{2}$ Ein Weg zu dieser Veränderung führt über Empathie, welche wir nicht nur im alltäglichen realen Leben, sondern auch beim Verstehen von fiktiven Charakteren in Romanen oder Filmen einsetzen. ${ }^{3}$ In den vergangenen Jahrzehnten ist eine Menge über Empathie und über deren Rolle in der Rezeption narrativer Fiktionen geschrieben worden: Empathie ist, grob gesagt, das Vermögen, die mentalen Zustände anderer nachzuvollziehen, das

1 Rorty, Richard: »Der Roman als Mittel zur Erlösung aus der Selbstbezogenheit«, in: Joachim Küpper/Christoph Menke (Hg.), Dimensionen ästhetischer Erfahrung, Frankfurt am Main: Suhrkamp 2003, S. 49-66, hier S. 52; vgl. auch ebd. S. 64.

2 Ebd. S. 56.

3 Vgl. neben Rorty auch Nussbaum, Martha: Upheavals of Thought. The Intelligence of Emotions, Cambridge: Cambridge University Press 2003. 
heißt Gefühle oder auch Wünsche und Überzeugungen zu verstehen. ${ }^{4}$ Empathie hat dann eine epistemische Funktion. Es kann ihr aber auch eine soziale und ethische Leistung zugesprochen werden, die darin besteht, dass sie unsere egoistische Aufmerksamkeit auf die Belange anderer lenkt. Oder anders gesagt: auf deren fremde Perspektive. Gerade fiktive Geschichten, so die These Rortys, aktivierten unser empathisches Vermögen, uns von uns selbst zu lösen. ${ }^{5}$ Aber was heißt es eigentlich, Perspektiven einzunehmen? Von welchen »Perspektiven « sprechen wir? Eine Perspektive ist technisch betrachtet das Verhältnis zwischen einem Blickpunkt und einem Objekt im Raum. Wir verwenden den Begriff aber auch metaphorisch und verstehen darunter eine wertende Sichtweise oder Haltung, zum Beispiel existenzieller, moralischer oder politischer Art. Alltagssprachlich sprechen wir ferner von »Handlungsperspektiven« im Sinne von Alternativen oder Optionen. Gerade bei einem Film - ich konzentriere mich hier auf den fiktiven Spielfilm - mit seinen vielseitigen audiovisuellen Techniken, seiner Narration und den Sichtweisen seiner Figuren liegt die Frage auf der Hand, was wir darunter verstehen, eine Perspektive »einzunehmen«, wenn es so viele Möglichkeiten gibt: Ist es die visuelle Perspektive? Ist es die Erzählperspektive? Ist es die Weltsicht der Figuren oder eines allwissenden Erzählers oder gar des Autors/Regisseurs? Können wir sogar von Perspektiven von Gegenständen und Körpern sprechen? Film ist nicht bloß Erzählung, sondern auch fotografierte, bewegte, vertonte, kadrierte, montierte Darstellung, nicht bloß Narration,

4 Vgl. für einen aktuellen Überblick z.B. die Einleitung in Coplan, Amy/Goldie, Peter (Hg.): Empathy. Philosophical and Psychological Perspectives, Oxford: Oxford University Press 2011.

5 Diese und andere Leistungen bescheinigen vor allem der Literatur auch unter anderen Martha Nussbaum (2003), Gottfried Gabriel (2014) und Catrin Misselhorn (2005; 2011). M. Nussbaum: Upheavals of Thought. The Intelligence of Emotions. Gabriel, Gottfried, »Fiktion, Wahrheit und Erkenntnis der Literatur«, in: Christoph Demmerling/Ingrid Vendrell Ferran (Hg.), Wahrheit, Wissen und Erkenntnis in der Literatur, Berlin: Akademie-Verlag 2014, S. 163-180. Misselhorn, Catrin: »Literatur, Wahrheit und Philosophie«, in: Catrin Misselhorn/Schamma Schahadat/Irina Wutsdorff (Hg.), Erkenntnis und Darstellung: Formen der Philosophie und der Literatur, Paderborn: Mentis 2011, S. 21-39. Dies.: »Ästhetische Erfahrung und die Perspektive der ersten Person«, in: Thomas Grundmann et al. (Hg.), Anatomie der Subjektivität. Bewusstsein, Selbstbewusstsein und Selbstgefühl, Frankfurt am Main: Suhrkamp 2005, S. 417-437. Auf die breite Diskussion der These des epistemischen und ethischen Werts der Empathie weist außerdem Alex Neill in seinem Aufsatz in diesem Band hin. 
sondern auch Expression ${ }^{6}$, und zwar, wie Vivian Sobchack schreibt, der Ausdruck von Erfahrung durch Erfahrung. ${ }^{7}$ Es gibt für RezipientInnen eine Pluralität an Möglichkeiten, auf verschiedene Perspektivenangebote des Films kognitiv, affektiv und leiblich zu reagieren.

Im Folgenden möchte ich den Zusammenhang von Perspektiven und solchen Reaktionen anhand einer Konzeption empathischer Resonanz in der filmästhetischen Erfahrung erläutern. »Resonanz« bezeichnet wörtlich ein »Wiederhallen« oder »Mitschwingen«, zum Beispiel eines Instrumentes beim Erklingen eines anderen Instrumentes oder eines Tons. Alltagssprachlich meinen wir mit Resonanz häufig so etwas wie ein »Feedback«. In der Philosophie und Psychologie wird der Begriff sowohl deskriptiv wie auch normativ verwendet für das intersubjektive und interkorporale Mitgehen des menschlichen Leibes in Anbetracht affektiver Vorgänge. ${ }^{8}$ Hinsichtlich »Perspektive « möchte ich einen weiten, integrativen Ansatz plausibilisieren, wonach eine Perspektive sowohl optisch als auch auditiv, sowohl körperlich, als auch figurativ zu verstehen ist. Ein Perspektivwechsel ist also nicht nur damit verbunden, dass etwas Neues sichtbar wird, wie es bei Rorty heißt, sondern zum Beispiel auch hörbar, spürbar und denkbar. Deshalb möchte ich auch von verschiedenen Formen von »Resonanz« sprechen, wie wir diese Perspektiven erfahren und auf sie antworten. Empathie ist ein Modus diverser, in der ästhetischen Erfahrung möglichen Resonanzmodi. Zur Resonanz zählen aber auch noch andere Einfühlungs- und Mitgefühlformen. Während der Begriff der Perspektiveneinnahme üblicherweise mit einem kognitiven Prozess in Verbindung gebracht wird, berücksichtigt »Resonanz « gerade die Leiblichkeit dieser Prozesse. ${ }^{9}$

6 Vgl. zu dieser »doppelten« Natur des Films auch Aumont, Jacques: »Der Point of View «, in: montage/av 16/1 (2007, frz. zuerst 1983), S. 13-44, hier S. 18.

7 »More than any other medium of human communication, the moving picture makes itself sensously and sensibly manifest as the expression of experience by experience«, Sobchack, Vivian, The Address of the Eye. A Phenomenology of Film Experience, Princeton: Princeton University Press 1992, S. 3.

8 Der Leib wird als »Resonanzraum« oder »Resonanzkörper « von Stimmungen und Gefühlen bezeichnet, vgl. Fuchs, Thomas: »Zwischen Leib und Körper«, in: Martin Hähnel, Marcus Knaup (Hg.), Leib und Leben. Perspektiven für eine neue Kultur der Körperlichkeit, Darmstadt: Wissenschaftliche Buchgesellschaft 2013, S. 82-93, hier S. 84.

9 »Resonanz« in einem normativ anspruchsvolleren Sinne ist zudem als ein substanzieller, ethischer Begriff für die gelungene Beziehung zwischen Ich und Welt zu konzeptualisieren, wie es Hartmut Rosa macht. Demnach wären zum Beispiel auch Anerken- 
Zunächst werde ich kurze Vorbemerkungen zum konzeptionellen Rahmen der Untersuchung machen. Im zweiten Abschnitt werde ich die Komplexität der Perspektiven im Film verdeutlichen. Am Beispiel der Einstiegssequenz von Michael Hanekes DAS WEISSE BAND werde ich den Perspektivenbegriff genauer erläutern und auf die verschiedenen Möglichkeiten von Perspektiven eingehen. Der dritte Abschnitt widmet sich vier verschiedenen Theoriegruppen zur Empathie, die ich anhand einer anderen Filmszene aus DAS WEISSE BAND diskutieren werde. Ich werde dort für einen phänomenologisch orientierten Empathiebegriff argumentieren, welcher eine imaginative Perspektiveneinnahme einerseits mit einer leiblichen Resonanz andererseits verknüpft. Der vierte Teil ist ein abschließendes Fazit.

\section{VORBEMERKUNGEN ZUR (FILM-)ÄSTHETISCHEN ERFAHRUNG}

Einen motivationalen Hintergrund der folgenden Überlegungen bildet die allgemeinere Fragestellung, was eigentlich die filmästhetische Erfahrung ausmacht. Dabei gehe ich davon aus, dass die Filmerfahrung mindestens durch zwei wichtige Parameter bestimmt ist: Erstens zeichnet sich Film durch eine Polyperspektivität aus audiovisuellen Techniken, Erzähltechniken und metaphorischen Perspektivierungen aus. Einige der Perspektivenangebote, die der Film macht - wie etwa die Kamera- und die Erzählperspektive - müssen wir allein schon aus filmund narrationsontologischen Gründen annehmen, um dem Film folgen zu können (wir können nicht die Augen verschließen, ohne etwas Wesentliches zu verpassen, noch können wir einem subjektiven Erzähler folgen, wenn es keinen gibt). Auch kann zum Beispiel eine Tragödie nicht einfach (ohne ironische Brechung oder künstlerische Sinnveränderung) als Komödie rezipiert werden. Zweitens erfasst uns Film mit seinen expressiven Eigenschaften affektiv und leiblich, so dass es zu kurz gegriffen wäre, das Verfolgen der Perspektiven auf kognitive Vorgänge zu reduzieren. Ferner ist der Begriff der »Erfahrung « als normativer und existenzieller Begriff zu verstehen; er meint nicht nur, dass wir in der ästhetischen Rezeption etwas erfahren (eine Geschichte kennen lernen), sondern et-

nungsbeziehungen Resonanzbeziehungen und Resonanz nicht bloß auf eine direkte affektiv-leibliche Wahrnehmung reduziert. Aus Platzgründen ist aber auf diese normative Erweiterung, die ausführlich begründet werden müsste, hier zu verzichten. Vgl. Rosa, Hartmut: Resonanz. Eine Soziologie der Weltbeziehung, Berlin: Suhrkamp 2016. 
was in einer Weise erfahren, welche besonders, reichhaltig und wertvoll ist, sogar eine »embodied and meaningful activity «. ${ }^{10}$ Wir machen mit Filmen wie mit anderen Kunstwerken eine Erfahrung, welche sich von nicht-ästhetischen Erfahrungen dahin gehend unterscheidet, dass sie zum Beispiel besonders konzentriert, intensiv, zeitlich gerahmt und ganzheitlich - das heißt leiblich und mental - ist. ${ }^{11}$ Einen solchen anspruchsvollen Begriff vertritt John Dewey, wobei wir solche Erfahrung Dewey zufolge nicht nur mit Kunst, sondern auch im Alltag erleben können. Er entwickelt »Erfahrung « unter anderem als einen abgerundeten (»rounded out «), zeitlich gegliederten und dynamischen Prozess mit einem Anfang, einem Höhepunkt und einem Abschluss. ${ }^{12}$ Nach Dewey erfahren wir das Objekt unserer ästhetischen Erfahrung (bzw. die Erfahrung selbst) immer in einem und aus einem Kontext heraus und im Verhältnis zu einer subjektiven Perspektive. Ein solcher Erfahrungsbegriff eignet sich meines Erachtens besonders gut für eine Untersuchung des Films. ${ }^{13}$ Meine These ist aber, dass das, was die filmästhetische Erfahrung ausmacht, auch mit den Erfahrungsperspektiven zusammenhängt, die wir empathisch vergegenwärtigen und so im imaginativleiblichem Nachvollzug neue Sichtweisen kennenlernen. ${ }^{14}$

10 V. Sobchack: The Address of the Eye. A Phenomenology of Film Experience, xvii. Vgl. zur philosophischen Diskussion von Erfahrung als existenziellem Begriff im Unterschied zu einem phänomenologischen und einem epistemologischem Begriff Deines, Stefan/Liptow, Jasper/Seel, Martin: »Kunst und Erfahrung. Eine theoretische Landkarte«, in Dies. (Hg.), Kunst und Erfahrung. Beiträge zu einer philosophischen Kontroverse, Berlin: Suhrkamp 2013, S. 7-37, hier S. 7 ff.

11 Damit ist nicht ausgeschlossen, dass wir solche Erfahrungen nicht auch in unserem alltäglichen Leben machen können. Im Anschluss an Dewey ist vielmehr davon auszugehen, dass ästhetische Erfahrungen nicht nur auf den klassischen Bereich des Ästhetischen (Kunst, Natur) zu beschränken sind, sondern dass wir z.B. auch im wissenschaftlichen Denken, beim Sport oder - ganz banal - beim Aufräumen ästhetische Erfahrungen machen können. »Ästhetisch « sind sie, wenn sie in besonderer Weise, wie oben angegeben, geschehen. Vgl. Dewey, John: Art as Experience, Carbondale, IL: Southern Illinois University Press 1989. [erstmals 1934].

12 J. Dewey: Art as Experience, S. 37 u. 47 ff.

13 Aus Platzgründen kann nicht die Frage beantwortet werden, ob die hier konstatierten Charakteristika der speziell filmästhetischen Erfahrung auch für allen anderen ästhetischen Erfahrungen in der gleichen Weise gelten.

14 V. Sobchack: The Address of the Eye. A Phenomenology of Film Experience, xix u. $95 \mathrm{f}$. 


\section{Der Begriff der Perspektive UND Die Perspektive(N) Des Films}

Das Drama DAS WeISSE BAND. EINE DEUTSCHE KINDERGESCHICHTE von Michael Haneke (DE 2009) beginnt mit einem Schwarzfilm, auf dem in weiß der Titel des Films geschrieben steht. Im Voice-Over teilt ein Mann mit, dass er von »seltsamen Ereignissen« eines Dorfes erzählen müsse, weil diese »möglicherweise auf manche Vorgänge in diesem Land ein erhellendes Licht werfen können. ${ }^{15}$ Der (extradiegetische) Erzähler, so stellt sich später heraus, ist in der Geschichte der Dorflehrer, dessen subjektive Perspektive er nun rückblickend wiedergibt. Er wird aber nicht von dem gleichen Schauspieler (Christian Friedel), sondern von einer anderen Person (Ernst Jacobi) gesprochen. Er wisse nicht, sagt er, ob die Geschichte in allen Details der Wahrheit entspreche und ob er sich immer richtig erinnere. Aber er sieht sich selbst offenbar in der Verantwortung, diesen Ereignissen nachzugehen und diese schließlich auch zu erzählen. Über die Länge der Erzählung in diesem ersten Bild wird langsam das nächste Bild aufgeblendet. Man sieht Wiesen, Felder, und aus der Tiefe des Raumes einen Mann auf einem Pferd herangaloppieren, während die Erzählerstimme weiter über dem Bild und dessen leisen O-Ton spricht: »...Begonnen hat alles, wenn ich mich recht entsinne, mit dem Reitunfall des Arztes. « ${ }^{16}$

Welche Perspektiven bieten uns die ersten Sequenzen des Films? Im Hinblick auf Film ist bei dem Begriff Perspektive zunächst an die optische Kameraperspektive zu denken: Der Standort und der Betrachtungswinkel der Kamera auf ein Objekt. »Point of View« oder »Point de Vue«, wie es passender im Englischen und Französischen heißt, ist der Punkt des Blickes, auf den und von wo der Blick fällt. ${ }^{17}$ Eine Kamera kann zum Beispiel ihr Objekt auf gleicher Höhe filmen und so die natürliche perspektivische Wahrnehmung imitieren. Beim Film sind die Kameraperspektiven häufig aber unnatürlich oder unrealistisch: Der Blickwinkel aus der Untersicht bzw. Froschperspektive etwa ist einer, den

15 Haneke, Michael: Das weiße Band. Eine deutsche Kindergeschichte. Das Drehbuch zum Film, Berlin: Berlin Verlag 2010, S. 7.

16 Ebd.

17 Vgl. J. Aumont: Der Point of View, S. 13 ff. Edward Branigan unterscheidet zwischen verschiedenen »Shots«, die eine optische Perspektive in Relation zu einer filmischen Figur jeweils unterschiedlich wiedergeben, je nachdem, wo sich die Kamera befindet: Etwa der Blick über die Schulter der Figur oder der subjektive Point of View Shot, Branigan 1992. Branigan, Edward: Narrative Comprehension and Film. London, New York: Routledge 1992. 
wir im alltäglichen Leben eher selten einnehmen. Auch die Einstellungsgröße des Kamerabildes offeriert Perspektiven: Eine Großaufnahme eines Gesichts etwa bietet uns eine andere Sichtweise an als eine Totale auf einen Raum. »Perspektive « ist also nicht nur im strengen Sinne als der Abstand und Winkel der Kamera zu den Objekten zu verstehen. Ich werde im Folgenden grob zwischen einer technischen und einer metaphorischen Verwendung des Begriffs der Perspektive unterscheiden. Unter der technischen lässt sich feiner zwischen der visuellen, auditiven, schnitt- und erzähltechnischen Perspektive differenzieren. ${ }^{18}$ Unter Perspektiven im metaphorischen Sinne fallen evaluative, ethische, politische, ideologische, handlungsoptionale Sichtweisen - wie wir die Welt wahrnehmen, beurteilen und uns in ihr bewegen - sowie affektive Sichtweisen in Form von Emotionen und Stimmungen, welche auch eine Weise sind, die Welt auf eine bestimmte Art wahrzunehmen. ${ }^{19}$ Der Begriff ist dem lat. perspicere entlehnt, dessen Bedeutung von »hindurchsehen« über »genau betrachten « bis hin $\mathrm{zu} »$ erkennen« reicht. Schon in unserer Alltagssprache verwenden wir diese verschiedenen Implikationen, wenn wir zum Beispiel davon sprechen, dass wir etwas in einem »anderen Licht betrachten« oder »aus einer anderen Warte «. Dies sind Metaphern dafür, dass wir etwas anders sehen bzw. erkennen, als wir es bislang gewohnt waren: Wir sehen durch das Gegebene hindurch auf etwas anderes. Das geschieht nicht in erster Linie dadurch, dass wir visuell eine andere Perspektive einnehmen, sondern indem wir unsere Imagination gebrauchen. ${ }^{20}$

Im Fall von DAS WEISSE BAND scheint nun aber das erste Bild, der Schwarzfilm mit dem Titel, aperspektivisch zu sein, denn aus einer Kameraperspektive ist es nicht gefilmt. Als aperspektivisch wäre das erste Bild jedoch nur zu bezeichnen, wenn wir »Perspektive« auf eine visuelle Sichtweise reduzierten. Wir hören aber auch eine Erzählstimme. Ohne schon den Inhalt der Erzählung zu be-

18 Vgl. etwa auch Francois Josts Unterscheidung zwischen Ocularisation (visuell, bildlogisch), Auricularisation (auditiv, tonlogisch) und Focalisation (narrativ, handlungslogisch); dieser Hinweis ist entnommen Schweinitz, Jörg/Tröhler, Margrit: »Editorial zum Themenschwerpunkt Figur und Perspektive (2)«, in: montage/av 16/1 (2007), S. 3-11, hier S. 7.

19 Vgl. zum Begriff der »Perspektive« in Kunst, Philosophie und Rechtswissenschaft die Texte im Sammelband von Koch, Gertrud (Hg.): Perspektive - Die Spaltung der Standpunkte. Zur Perspektive in Philosophie, Kunst und Recht, München: Fink 2010. Außerdem Schulte-Sasse, Jochen: »Perspektive/Perspektivismus« (= Ästhetische Grundbegriffe, Band 4), Stuttgart/Weimar: Metzler 2003, S. 758-778.

20 Vgl. Scruton, Roger: Art and Imagination. A Study in the Philosophy of Mind, London: Methuen 1974. 
rücksichtigen, können wir hier eine Erzählperspektive ausmachen, die uns auditiv vermittelt wird: Wir hören synchron zum Bild etwas über den Standpunkt eines Mannes: Sein Standort in der Geschichte und seine Sicht auf zurückliegende Geschehnisse. Indem hier jemand von vergangenen Ereignissen spricht, an die er sich nicht in allen Details erinnert, wird deutlich, dass eine Perspektive auch existenziell, kontextual und zeitlich an einen Standort gebunden ist; sie ist persönlich und subjektiv. Als geübte ZuschauerInnen begreifen wir sehr schnell, dass das, was erzählt wird, aus einer subjektiven Perspektive und vor einem historischen Hintergrund erzählt wird. Dies wird durch zwei ästhetische Mittel verstärkt: Erstens wirken das erste Schwarz- und die darauffolgenden SchwarzweißBilder historisch und anti-naturalistisch: Sie betonen die subjektive Erinnerung des Erzählers, indem sie als Schwarzweiß-Bilder den Artefaktcharakter der Geschichte betonen und damit die, wie Haneke selbst es formuliert, »Wahrheit oder Wirklichkeit der Geschehnisse « in Zweifel ziehen lassen. ${ }^{21}$ Dies verweist zudem auf eine extradiegetische Metaperspektive: Aumont nennt dies die mentale Haltung, die die Darstellung der Figuren oder des filmischen Ganzen überformt. ${ }^{22}$ Den zweiten Hinweis für eine zeitliche Diskrepanz zwischen Erzählinstanz und Erzähltem gibt die Stimme des Sprechers, wobei wir dies erst erkennen, wenn der Erzähler aus dem Off sich als intradiegetische Figur in der Geschichte erweist. Die Erzählerstimme klingt älter als die Stimme der Figur. So können wir dem Erzähler zwei Perspektiven zuordnen, einmal jene, die er einnahm, als er damals als Dorflehrer Zeuge »mancher Vorgänge « wurde, zum anderen jene, die er in der extradiegetischen Gegenwart rückblickend und reflektierend - aus der Perspektive eines alten Mannes - einnimmt. Die Perspektivenvielfalt geht aber noch weiter: Der Erzähler richtet sich mit seiner Geschichte an eine fiktive und eine reale Zuhörerschaft, deren Sicht auf Ereignisse - wieder ein alltagssprachliches Synonym - sich möglicherweise durch die Erzählung verändert: Es würde eventuell etwas »Neues«, um auf Rorty zurückzukommen, sichtbar werden. Auch dass es überhaupt diesen Erzähler gibt, ist ein perspektivierendes Mittel:

21 In einem Interview vertritt Haneke die These, Farbe provoziere eine »verlogene Illusion von naturalistisch wiedergegebener Wirklichkeit, »sie tut so als wüßten wir wie es damals war. Und niemand weiß wirklich, wie es damals war. Das Schwarzweiß unterstreicht den Artefakt-Charakter.«, M. Haneke: Das weiße Band. Eine deutsche Kindergeschichte. Das Drehbuch zum Film, S. 147. Vgl. auch Cieutat, Michel/ Rouyer, Philippe: Haneke über Haneke, Berlin/Köln: Alexander Verlag 2013 (frz. zuerst 2012), S. 313.

22 J. Aumont: Der Point of View; vgl. außerdem J. Schweinitz/M. Tröhler: Editorial zum Themenschwerpunkt Figur und Perspektive (2), S. 8. 
Es unterstreicht die wertende Position des Films, denn der Erzähler schreibt sich selbst eine evaluative Funktion zu, indem er die Ereignisse im Rückblick interpretierend berichtet, weil er meint, sie berichten zu müssen, da sie Erklärungspotenzial haben. ${ }^{23}$ Ich habe eben bereits die ästhetischen Mittel angesprochen: Ein Schwarzweiß-Bild ist nicht nur narrativ unterstützend, es hat auch eine eigenständige, expressive Qualität, deren Expression sich von einem Farbbild zum Beispiel darin unterscheidet, dass eine Distanz hergestellt wird, weil auf etwas Altes, Vergangenes verwiesen wird. Es vermittelt aber auch eine bestimmte Stimmung und Atmosphäre. Auch dies können wir als Perspektive begreifen. Denn Stimmungen sind, wie angedeutet, selbst Weisen, die Welt wahrzunehmen. ${ }^{24}$ In einer depressiven Stimmung haben wir eine andere Perspektive auf die Welt als in einer leichten, gehobenen, lustigen Stimmung. Eine Stimmung der Angst ist mit der Perspektive auf eine Situation als bedrohlich konstitutiv verknüpft usw.

Wie bei dieser knappen Darstellung deutlich werden sollte, sind Perspektiven im Film nicht auf die visuelle Kamera noch auf die narrativen, handlungslogischen Perspektiven der fiktiven Figuren beschränkt. Gerade der Film weist eine komplexe Perspektivenvielfalt auf. Denn beim Film greifen technische, erzählerische und metaphorische Perspektiven visuell, auditiv, editorisch ineinander und machen in ihrer Beziehung untereinander die vielschichtige filmästhetische Erfahrung aus. Ihnen jeweils innewohnen können verschiedene Affizierungsprozesse: So wird etwa eine narrative Perspektive durch eine ausgedrückte Emotion oder Stimmung der Figur begleitet, die Metaperspektive durch eine filmischexpressive Atmosphäre zum Ausdruck gebracht (ein Film Noir hat eine andere Atmosphäre als eine zeitgenössische konventionelle Liebeskomödie), und die technischen Perspektiven können ihrerseits Mittel sein, die verschiedenen Arten von Affekten der Erzählung zu unterstützen. ${ }^{25}$

$23 \mathrm{Zu}$ solchen Erzählerinstanzen vgl. Nünning, Ansgar: »Die Funktion von Erzählinstanzen. Analysekategorien und Modelle zur Beschreibung des Erzählerverhaltens«, in: Literatur in Wissenschaft und Unterricht 30 (1997), S. 323-349., S. 336.

24 Wellbery, David: Stimmung (=Ästhetische Grundbegriffe, Band 5), Stuttgart/Weimar: Metzler 2003, S. 703-733.

$25 »$ Affekt « wird hier als Oberbegriff für alle affektiven Phänomene wie Emotionen oder Stimmungen verwendet. Der Begriff der Atmosphäre ist eng mit dem der Stimmung verwandt. Gernot Böhme, der den Begriff in der philosophischen Diskussion als ein Grundbegriff einer neuen Ästhetik stark geprägt hat, beschreibt »Atmosphäre« als eine stimmungshafte Eigenschaft einer räumlichen Umgebung, deren Ausstattung und Beziehung zum leiblich anwesenden Subjekt, vgl. Böhme, 2013. Der Phänomenologe 
Zwischen Perspektiven des Films, im Film und beim Betrachten eines fiktiven Films auf der einen Seite und den Perspektiven in nicht-ästhetischen oder nicht-fiktionalen Kontexten gibt es einige wichtige Unterschiede: Was die Perspektiven im Film bzw. unsere Zuschauerrezeption dieser Perspektiven betrifft, sind wir nämlich zugleich freier und eingeschränkter. Eingeschränkter sind wir, da der Film als narratives, fiktionales Konstrukt und als Ausschnitt uns Perspektiven vorgibt. Während wir in der Realität einer Vielzahl von Möglichkeiten ausgesetzt sind, unser Umfeld aus der einen oder anderen Perspektive sehen zu können, indem wir faktisch oder metaphorisch unseren Standort ändern, ohne dass uns stets jemand vorgibt, wohin wir unseren Blick wenden sollen, wird unser Blick auf die fiktiven Figuren »vorperspektiviert $« .{ }^{26}$ Wir wissen nur das über sie oder erfahren nur jenes über sie, was uns der Film und der Filmemacher über die Narration und Expression erfahrbar macht und was wir auf dieser Grundlage imaginativ ergänzen. ${ }^{27}$ Carroll spricht hierbei von einem kriteriellen Vorfokussieren: Das, was in Bild und Erzählung dargestellt wird, ist so ausgewählt, dass es den Fokus der ZuschauerInnen auf bestimmte Details richtet, die bestimmte emotionale Regungen und moralische Urteile evozieren. ${ }^{28}$ Die Kameraeinstellungen und andere expressive Mittel bestimmen dabei die narrative Perspektive konstitutiv mit. ${ }^{29}$ Solche starken und eindeutigen Vorperspektivierungen haben

Hermann Schmitz definiert »Gefühle als räumlich ergossene, leiblich ergreifende Atmosphären« und Atmosphären als Besetzungen eines flächenlosen Raums. Vgl. Schmitz, Hermann: Der Leib, der Raum und die Gefühle. Bielefeld: Edition Sirius 2007.

26 J. Schweinitz/M. Tröhler: Editorial zum Themenschwerpunkt Figur und Perspektive (2), S. 3. Das trifft auch auf den Dokumentarfilm zu, der über seine Erzählstruktur, Kameraführung etc. die Perspektivierungen ebenfalls lenkt. Ich konzentriere mich hier aber auf den fiktiven, narrativen Film.

27 Das gehört nicht zuletzt auch zu der manipulativen Kraft, die Film haben kann.

28 Carroll, Noël: »On some Affective Relations between Audiences and the Characters in Popular Fictions«, in: Amy Coplan/Peter Goldie (Hg.), Empathy. Philosophical and Psychological Perspectives, Oxford: Oxford University Press 2011, S. 162-184, hier S. 169. Vgl. auch Carroll: »Film, Emotion, and Genre«, in: Carl Plantinga/Greg M. Smith (Hg.), Passionate Views, Baltimore, MD: Johns Hopkins University Press 1999, S. 21-47, hier S. 29: »[...] the filmmakers have already done much of the work of emotionally organizing scenes and sequences for us through the ways in which the filmmakers have foregrounded what features of the events in the film are salient."

29 Vgl. J. Schweinitz/M. Tröhler: Editorial zum Themenschwerpunkt Figur und Perspektive (2), S. 5. 
wir in der Wirklichkeit jenseits von Fiktionen nicht. ${ }^{30}$ Freier aber sind wir in zweierlei Hinsicht: Da wir uns auf diese vorgegebenen Perspektiven konzentrieren können, müssen wir erstens keine ablenkende Kontextualisierungs- und $\mathrm{Zu}$ ordnungsarbeit leisten, sondern können uns ganz auf die ästhetische Erfahrung die eine Art Spiel ist - einlassen. Denn eine Narration schafft mit ihren Vorperspektivierungen und Lenkungen zugleich auch Strukturierung, Einordnung und Distanz. ${ }^{31}$ Zudem sind wir ethisch freier, das heißt entlastet, was unsere Handlungsmöglichkeiten und -pflichten betrifft, da wir in das Geschehen nicht eingreifen können. Wir können Perspektiven ausprobieren, ohne dass dies von uns ein direktes Handeln in ethischer Hinsicht erfordert. ${ }^{32}$ Und schließlich macht uns eine Perspektiveneinnahme auch in existenzieller Hinsicht freier, indem uns neue Möglichkeiten des Seins eröffnet werden. ${ }^{33}$

Die Perspektivenmöglichkeiten der Wirklichkeit - in welchem Lichte wir jemanden betrachten können - sind hingegen nur scheinbar freier, denn sie sind auch diffuser, episodischer und fragmentarischer, ${ }^{34}$ vielfach scheitern wir, da wir zu wenig über die Menschen, die uns im alltäglichen Leben begegnen, wissen. Vielmehr müssen wir uns dieses Wissen mühsam erarbeiten und wir können nicht mit den Perspektiven unserer Umgebung spielen. Wir müssen jemanden genau kennen lernen, um seine oder ihre Perspektive - zum Beispiel auf eine politische Frage oder auf das Leben als Ganzes - verstehen zu können. Im Gegensatz zu unserem Umgang mit Fiktionen sind wir dabei auch ethisch gefordert, zu handeln, wenn wir eingreifen können.

30 Es gibt auch in der Wirklichkeit Fiktionalisierungen und fiktionale, narrative Zusammenhänge, etwa bei Stadtführungen, die lenkend die Geschichte einer Stadt erzählen. Vgl. zum Verhältnis von Realität und Fiktion Iser, Wolfgang: Das Fiktive und das Imaginäre, Frankfurt am Main: Suhrkamp 1991.

31 Vgl. Breyer, Thiemo: Verkörperte Intersubjektivität und Empathie, Frankfurt am Main: Vittorio Klostermann 2015, S. 262.

32 »In entering a fictional world, we are exercising our feelings, but not acting from them «, Scruton, Roger: The Aesthetics of Music, Oxford: Clarendon Press 1997, S. 355, Herv. i.O..

$33 »[\ldots]$ the possibilities of becoming other than what is«, V. Sobchack: The Address of the Eye. A Phenomenology of Film Experience, xix.

34 Vgl. dazu auch die Unterscheidung zwischen alltäglicher und virtueller Erfahrung von Vendrell Ferran, Ingrid: »Das Wissen der Literatur und die epistemische Kraft der Imagination«, in: Christoph Demmerling/Ingrid Vendrell Ferran: Wahrheit, Wissen und Erkenntnis in der Literatur. Philosophische Beiträge, Berlin: De Gruyter 2014, S. 119-140, S. 129. 
Haben auch Gegenstände, nicht-menschliche Entitäten und Körper im Film Perspektiven? Wir können davon ausgehen, dass auch die »Dinge im Film« anders wahrgenommen werden als in nicht-ästhetischen Zusammenhängen: Gerade da der Film mit seiner Kameraperspektive und seinem Narrativ Vorperspektivierungen schafft, erhalten die Dinge eine Bedeutung oder werden als etwas »behandelt«, wie Cavell schreibt, was sie in Wirklichkeit nicht sind. ${ }^{35}$ Gegenstände, so unscheinbar sie erscheinen mögen, haben im Film ihren Platz, ihre Rolle. ${ }^{36}$ Cavell betont, dass hierfür die Imagination und der Perspektivenwechsel wichtig sind, um Dinge als etwas anderes wahrzunehmen. Auch Landschaften, Natur, Bäume, Meer, Straßen, Häuser etc. sind im Film nicht bloße Requisiten, sondern drücken Stimmungen, Atmosphären aus, unterstützen insofern eine Metaperspektive oder Figurenperspektiven oder sind selbst als Perspektiven zu verstehen. Körper können als Perspektiven bestimmt werden, wenn wir davon ausgehen, dass wir a) als menschliche Wesen, die sich in der Welt bewegen, immer mit einem Körper in der Welt sind und diese wahrnehmen, b) die Welt auch an unserem Körper empfinden: So fangen wir etwa an zu zittern oder wollen fliehen, wenn wir einer Bedrohung ausgesetzt sind, uns c) körperlich ausdrücken, wenn wir unser Fühlen und Denken vermitteln und d) kognitive Prozesse nicht von unserem leiblichen In-der-Welt-Sein zu trennen sind. Die Aspekte a-d werden vor allem in phänomenologischen Ansätzen und jüngst in Theorien einer Philosophie der Verkörperung innerhalb der Kognitionswissenschaften vertreten. ${ }^{37}$

Die hier bloß knappe Darstellung der Polyperspektivität des Films und der Rezeption ist nicht als rein deskriptiv zu verstehen, sondern als normativ: Perspektiven werden in der filmästhetischen Erfahrung angeboten, und die FilmzuschauerInnen sind aufgefordert, ihrerseits diese Angebote aufzugreifen, um dem Film kognitiv und affektiv folgen zu können. Das damit notwendig unterstellte

35 Cavell, Stanley: »Was wird aus den Dingen im Film«, in: Dimitri Liebsch (Hg.), Philosophie des Films. Grundlagentexte, Paderborn: Mentis 2006 [engl. zuerst 1978], S. 100-110, hier S. 102.

36 Vgl. dazu etwa die metaphorische und handlungslogische Rolle der Gegenstände im Film AMOUR (F/D/A 2012) von Michael Haneke. So wurden zum Beispiel die Bücher in den Regalen in der Wohnung der Hauptfiguren alphabetisch und nach Thema geordnet, obwohl man in keiner Einstellung die Titel auf den Büchern lesen kann. Haneke ist der Überzeugung, dass man solche Details dennoch »in den Bildern spürt«, M. Cieutat/P. Rouyer: Haneke über Haneke, S. 355.

37 Fingerhut, Joerg/Hufendiek, Rebekka/Wild, Markus (Hg.): Philosophie der Verkörperung. Grundlagentexte zu einer aktuellen Debatte, Berlin: Suhrkamp 2013, S. 9. 
Interesse an den Perspektiven des Films ist nicht nur instrumentell, sondern auch darin begründet, dass wir mit der Perspektiveneinnahme einen epistemischen und ethischen Wert verbinden und darin auch den Gesamtwert der ästhetischen Erfahrung mit Kunstwerken bzw. Film sehen können: Indem wir andere Perspektiven einnehmen, lernen wir Neues kennen, erweitern unser Wissen, unseren Horizont und gelangen möglicherweise, wie es Rorty vorschwebt, zu mehr moralischer Reife und Autonomie. ${ }^{38}$ Dazu bedarf es mindestens eines menschlichen Vermögens, dem ich nun den zweiten Teil dieses Aufsatzes widmen möchte, nämlich der Empathie. Sie ist es, die wir einsetzen müssen, wenn wir Perspektiven nachvollziehen wollen, was Bedingung ist, um dem Film folgen zu können.

\section{EMPATHIE}

Wenn wir in der Rezeption einem Film in seiner Ästhetik und Handlungslogik folgen wollen, so gehört dazu ein integrales Interesse, mehr über die Figuren und deren Wünsche, Überzeugungen, Emotionen, Stimmungen, Handlungen und Handlungsmotive etc. zu erfahren, um verstehen und beurteilen zu können, warum sie wie denken, fühlen und handeln. Nur so bleiben wir überhaupt »am Ball «. ${ }^{39}$ Folgen können wir dem Film und seinen Figuren, so meine These, indem wir Perspektiven einnehmen. Dies ist freilich nicht nur so gemeint, dass wir die Kameraperspektive visuell einnehmen, sondern vor allem, dass wir uns die Sichtweisen des Films und der Figuren zu vergegenwärtigen versuchen. Dazu verhilft uns unsere Fähigkeit der Empathie.

Empathie bedeutet, allgemein gesagt, die mentalen Zustände anderer Personen oder Figuren, vor allem deren komplexe Emotionen und Überzeugungen, nachzuvollziehen und zu verstehen.

Ich unterscheide grob vier größere verschiedene Theoriegruppen, innerhalb derer jeweils verschiedene Ansätze unterschieden werden: Zur ersten Gruppe zähle ich Ansätze, welche Empathie als Nachahmung, Ansteckung oder Spiegelung definieren. Unter Empathie wäre dann der mimetische, präreflexive Vorgang des Mitgehens auf affektiver Ebene zu verstehen, was Kritiker einer solchen Konzeption zu Recht begrifflich als »Ansteckung« von Empathie unterscheiden. Da diese Theoriegruppe aber zugleich bedeutend für die Empathiedis-

38 R. Rorty: Der Roman als Mittel zur Erlösung aus der Selbstbezogenheit.

39 Gertrud Koch nennt dies die »und dann? und dann...? «-Erwartung, die ZuschauerInnen und LeserInnen an Geschichtenerzähler haben, vgl. Koch, Gertrud: Breaking Bad, Zürich/Berlin: Diaphanes 2015, S. 12. 
kussion ist, soll sie hier auch unter dem Begriff der Empathie und der basalleiblichen Resonanz vorgestellt werden. Zur zweiten Gruppe gehört die Theory Theory, nach welcher wir nach einem volkspsychologischen Modell auf die mentalen Zustände Anderer inferentiell schließen. Die dritte Gruppe bildet die Simulationstheorie, wonach wir uns imaginativ in die Lage des Anderen hineinversetzen und die entsprechenden Zustände simulieren. Unter die vierte Gruppe fallen phänomenologisch geprägte Ansätze, welche Empathie in erster Linie als direkte Wahrnehmung des Ausdrucksverhaltens anderer verstehen. Ich subsumiere meinen eigenen Ansatz unter diese Gruppe, allerdings verknüpfe ich dabei das Kriterium der leiblichen Ausdruckswahrnehmung mit dem der imaginativen Perspektiveneinnahme.

Ich möchte zur Veranschaulichung der verschiedenen Empathietheorien wieder auf das Filmbeispiel DAS WEISSE BAND zurückkommen, dieses Mal aber eine Szene im ersten Drittel des Films analysieren, welche im Haus des strengen Pfarrers spielt, der seine Kinder autoritär erzieht. In einer einzigen langen Einstellung folgt die Kamera zunächst der Pfarrersfrau, die zwei ihrer Kinder, Martin und Klara, in ihren Zimmern abholt und mit ihnen zum Esszimmer geht. Die visuelle Perspektive der Kamera ist darauf gerichtet, wie die Mutter die Tür öffnet. Wir sehen den Pfarrer und die anderen Kinder am Tisch sitzen. Die Mutter, Martin und Klara gehen in den Raum. Die Tür wird geschlossen. Die Kamera verharrt unbeweglich in einigem Abstand, so dass der lange Flur zu sehen ist, an dessen Ende die verschlossene Tür ist. Das Bild enthält wichtige handlungsbezogene sowie extradiegetische, selbst-reflexive Implikationen: Die Kadrierung ist akkurat und geometrisch, ein von Haneke häufig verwendetes Stilmittel, das die filmisch selbstreferentielle Rahmen- und Türsymbolik des Innen und Außen widerspiegelt: Als ZuschauerInnen sind wir in ontologischer Hinsicht grundsätzlich vom Geschehen auf der Leinwand abgeschirmt. Cavell verwendet dafür den doppeldeutigen Begriff des »screened $\ll$ : Ein Film ist auf die Leinwand (»screen «) projiziert und schirmt uns davon zugleich ab, denn wir können nicht selbst in den Film durch die Leinwand hindurch in das Filmgeschehen hineinsteigen. »The world of a moving picture is screened [...] What does a silver screen screen? It holds me from the world it holds - that is, makes me invisible. $\ll^{40}$ In der Szene des Haneke-Films wird dies aufgegriffen; wir bleiben wie ein unbeobachteter Beobachter draußen. Wir sehen aber nun distanziert aus der Kameraperspektive, wie Martin den Raum verlässt und mit einem Rohrstock zurückkehrt. Anschließend hören wir die Stimme des Vaters und dann in regelmä-

40 Cavell, Stanley: The World Viewed. Reflections on the Ontology of Film. Enlarged Edition, Cambridge, MA: Harvard University Press 1979 (erstmals 1971), S. 24. 
Bigen Abständen das Geräusch der Rohrstockschläge sowie Martins Schreie. Zur visuellen Perspektive kommt hier entscheidend der Ton hinzu, der über die Geräusche und Stimmen aus dem verschlossenen Raum eine eigene Perspektive wiedergibt. Wie reagieren wir in dieser Szene empathisch? Meines Erachtens gibt es entsprechend der vier Theoriegruppen auch vier verschiedene Möglichkeiten, auf diese Situation zu reagieren. Ob wir jede dieser Möglichkeiten tatsächlich Empathie nennen sollen, wird im Folgenden zur Diskussion stehen.

1. Nach der Nachahmungstheorie wäre anzunehmen, dass wir beim Hören der Schreie zusammenzucken und den Schmerz oder zumindest das damit verbundene Leid, die Angst etc. automatisch mitempfinden. Traditionell liegt dieser Theorie Theodor Lipps' Einfühlungsbegriff zugrunde, der Empathie als instinktiven Vorgang der Gefühlsnachahmung und automatischen Projektion beschreibt. ${ }^{41}$ Die einfühlende Person spiegelt in dem Fall die mentalen Aktivitäten oder Erfahrungen Anderer auf der Grundlage der Beobachtung körperlicher Akte oder Expressionen (wie Gestik, Mimik). Es kommt dann mindestens zu einer Tendenz, die beobachteten Abläufe körperlich zu imitieren, zum Beispiel beim Anblick eines verärgerten Gesichts selbst ein solches Gesicht zu machen. Gerade weil hier von einer Spiegelung gesprochen wird, kann auch die seit den 1990er Jahren viel diskutierte Theorie der Spiegelneuronen unter diese Kategorie zusammengefasst werden. ${ }^{42}$ Nach dieser Theorie werden, kurz gesagt, beim Anblick einer Emotion eines Anderen spiegelhaft die gleichen Neuronen aktiviert wie wenn wir selbst diese Emotion originär spüren. Ferner gehört zu dieser Gruppe das Konzept der emotionalen »Gefühlsansteckung«, welches das Phänomen einfängt, dass wir von Gefühlen anderer reflexartig infiziert werden können, zum Beispiel, wenn wir in eine Menschenmenge geraten, die aggressiv aufgeladen ist. In allen diesen Fällen ist der Vorgang ein unbewusster, bei dem Gefühle einer anderen Person automatisch angenommen werden.

41 Vgl. Lipps, Theodor: Leitfaden der Psychologie, Leipzig: Wilhelm Engelmann 1903, S. 713-719.

42 Vgl. z.B. Rizzolatti, Giacomo/Fadiga, Luciano/Fogassi, Leonardo/Gallese, Vittorio: »Resonance Behaviour and Mirror Neurons«, in: Archives Italiennes de Biologie Nr. 137 (1999), S. 85-100. Für einen Überblick vgl. auch Goldman 2006. Stueber spricht im Zusammenhang mit den spiegelneuronalen Mechanismen von einer »basalen Empathie«. Vgl. Stueber, Karsten R.: Rediscovering Empathy, Agency, Folk Psychology, and the Human Sciences, Cambridge: MIT Press 2006. Um dies von Empathie im anspruchsvollen Sinne besser abzusetzen, schlage ich vor, von einer Form basaler neuronaler Resonanz, die sich leiblich ausdrückt, zu sprechen. Die Spiegelneuronentheorie wird häufig auch unter die Gruppe der Simulationstheorie subsumiert. 
Gerade den Film betreffend, scheint diese Theorie einiges an Plausibilität zu besitzen: Die Erfahrung mit fiktiven, narrativen Filmen wird häufig auch als »immersiv « bezeichnet. ${ }^{43}$ Der Begriff der Immersion meint, dass wir in das Filmerlebnis eintauchen, impliziert eine Selbst- und Weltvergessenheit und ist verbunden mit einer Art Überwältigung durch das filmische Geschehen. ${ }^{44}$ Der mimetische Einfühlungsbegriff nach Lipps scheint zur Beschreibung dieses Phänomens genau das geeignete terminologische Werkzeug: Wenn wir an unsere Reaktionen in schnell geschnittenen und einem mit uns buchstäblich einlullenden »Surround Sound « versehenen Action-Filmen denken, so sind diese genau so zu beschreiben: Wir bleiben dabei nicht konzentriert verhalten im Kinosessel sitzen, sondern schrecken unwillkürlich zurück, sinken ein, spüren unser Herz rasen etc. Aber nicht nur der naheliegende Action-, auch der ruhige ArthouseFilm kann solche immersiven Prozesse in Gang setzen, wenn er uns mit langen Einstellungen in eine ruhige oder gespannte Stimmung versetzt. ${ }^{45}$ Auch für die Erklärung des Einflusses der expressiven Mittel ist Lipps Einfühlungstheorie hilfreich: Nach Lipps geschieht die Imitation auch mit Gegenständen, zum Beispiel Steinen, Bäumen etc. Wir ahmen dann deren Tendenz nach, zum Beispiel die Abwärtsbewegung eines Steins, der einen Berg hinabrollt. ${ }^{46}$ Ein akkurat kadrierter Kameraausschnitt mit Schwarzweiß-Bildern könnte demnach in uns ein Gefühl der Strenge, Ordnung, Präzision, Kühle und Distanz hervorrufen. Es ist eine leibliche Resonanz, also ein Mitschwingen oder Widerhallen auf die Atmosphäre oder die Stimmung des Bildes. Obwohl dies ein reflexhafter Vorgang ist, können wir auch auf diese Weise Perspektiven wahrnehmen, nämlich leiblich. Indem wir Stimmungen kontagiös erfassen bzw. sie uns, erfassen wir auch die damit verbundenen Perspektiven, die wir uns zwar nicht bewusst vergegenwärtigen, die wir aber am eigenen Leib spüren: Ein stimmungshaftes In-der-WeltSein ist auch eine Weise, die Welt wahrzunehmen.

43 Vgl. Curtis, Robin: »Immersion und Einfühlung«, in: montage/av,17/2 (2008), S. 89107.

44 Ebd. $89 \mathrm{f}$.

45 Ein Beispiel wäre der dreistündige, fast vollständig auf Sprache verzichtende Dokumentarfilm DiE GROSSE STILLE (F/CH/D 2005) von Philip Gröning über das Schweigekloster Grande Chartreuse.

46 Vgl. T. Lipps: Leitfaden der Psychologie, S. 193. Vgl. Voss, Christiane: »Einfühlung als epistemische und ästhetische Kategorie bei Hume und Lipps«, in: Robin Curtis/Gertrud Koch (Hg.), Einfühlung. Zu Geschichte und Gegenwart eines ästhetischen Konzepts, Paderborn/München: Fink 2009, S. 31-47, hier S. 40. 
Wie aber sollen wir wissen, dass es sich um eine neue Perspektive handelt und vor allem warum diese Perspektive besteht? Sind wir überhaupt so distanzlos und »verloren« im Kino, wie es Immersions- und Einfühlungstheorien nach Lipps nahelegen $?^{47}$ Die Konzeption von Lipps wurde häufig als eine egozentrische Konzeption kritisiert, wonach die Rezipientin nur weiß, was sie selbst fühlt und dies auf den eigenfühlten Gegenstand oder die Person projiziert. ${ }^{48}$ Auch scheint es in der beschriebenen Haneke-Szene besonders schwierig, von einer direkten Nachahmung zu sprechen, da die Kamera in Distanz zu dem Geschehen tritt, das wir dann nur noch hören, während der Empathie-Vorgang nach Lipps eine optische Wahrnehmung der Bewegungen, Gestiken, Mimiken etc. Anderer voraussetzt.

Ein zentrales Problem dieser Konzeption im Hinblick darauf, wie wir andere verstehen, besteht unter anderem darin, dass bei der Ansteckung oder Nachahmung das Gefühl unreflektiert übernommen wird, ohne dass man über dessen Ursachen, Objekte und Konsequenzen im Klaren ist. Die Gefühle des Anderen werden hier nicht verstehend nachvollzogen, sondern infektiös übernommen. Lipps' Ansatz ist ein gänzlich anti-hermeneutischer Ansatz, bei dem es in der ästhetischen Erfahrung gerade nicht um Verstehensbemühungen geht. ${ }^{49}$ Kognitives Urteilen und Fühlen fallen bei ihm auseinander. Für eine Ästhetik, welche einen epistemischen und ethischen Wert von Perspektiveneinnahmen in der Ästhetik sowie eine Verbindung von Leib und Geist, Denken und Fühlen annimmt, eignet sich dieser Ansatz nicht. ${ }^{50}$ Ein weiteres Problem, das bereits angedeutet wurde, ist, dass beim Lipps'schen Ansatz die Distanz zwischen Rezipientin und eingefühlter Figur gerade verloren gehen soll und es dann nicht mehr deutlich ist, dass es die Figur ist, welche fühlt. Gerade zu einer ästhetischen Erfahrung gehört aber auch eine Distanz, damit sie sich von einer rein lustvollen Konsumation unterscheidet. Haneke wendet in der beschriebenen Szene ein sehr starkes Distanzierungs- und Reflexionsmittel an, indem die Kamera wie ein unbeteiligter Beobachter außen vor bleibt und wir das Geschehen nur auf auditiver Ebene mitgeteilt bekommen anstatt zum Beispiel aus der Perspektive »über der Schulter « der beobachtenden Mutter oder aus dem subjektiven Point of View des schlagenden

47 Voss, die zwar eine Illusionsästhetik vertritt, bestreitet das und wendet sich kritisch gegen jene Immersionstheorien, welche Immersion und Reflexion/Distanz als Gegensatz verstehen. Vgl. Voss, Christiane: Der Leihkörper. Erkenntnis und Ästhetik der Illusion, Paderborn: Fink 2013, S. 184.

48 Ebd. S. $205 \mathrm{ff}$.

49 Ebd. S. 205 ff., insb. S. 210 f.

$50 \mathrm{Vgl}$. für einen anti-reduktionistischen Versöhnungsansatz auch ebd. 
Vaters. Wie veranschaulichen wir uns das, was hinter der Tür vorgeht? Reicht dazu der Hörsinn aus, um sich das Ganze der Situation zu vergegenwärtigen? Und schließlich besteht ein Problem darin, dass wir als RezipientInnen oft gar nicht dasselbe fühlen wie die Figur, ja gerade nicht fühlen müssen und dennoch (oder gerade dann) verstehen, in welcher Situation sie sich befindet. Nach dem Nachahmungsansatz würden wir aber bei jeder Gestik und Darstellung von Gefühlen diese automatisch imitieren. Noël Carroll hat dagegen berechtigterweise vermehrt Kritik vorgebracht. ${ }^{51}$ Die Gefühle einer Filmfigur und die der Zuschauerin sind nämlich sehr häufig asymmetrisch und sollen es auch sein. Zum Beispiel können wir uns um die Figur Martin sorgen, während er gerade schreit - er selbst sorgt sich möglicherweise gar nicht um sich, sondern kann sich nur auf seine Schmerzen konzentrieren. Wir aber fürchten etwa um das Leben der Figur, haben Mitleid mit ihm und empfinden Wut auf den Vater. ${ }^{52}$ Unsere Emotion ist dann ein sympathetisches Mitgefühl, nicht ein mimetisches gleiches Fühlen.

Statt von Empathie sollte man in den Fällen der Nachahmung, Spiegelung und Ansteckung daher von einem passiven, unbewussten Mitfühlen sprechen. Es ist nicht mit der komplexen Empathie als Verstehensprozess der Emotionen und anderen mentalen Prozesse gleichzusetzen. ${ }^{53}$ Mit der Kritik an diesen Modellen soll aber nicht der Vorgang der Ansteckung, Spiegelung usw. als solcher zurückgewiesen werden - denn natürlich gibt es diese Erfahrung gerade beim Film. Wie oben angegeben, haben wir es dabei durchaus mit einer sehr basalen, primitiven Form der Resonanz zu tun, die auch ein konstitutiver Teil der Filmerfahrung ist. Worauf es mir an dieser Stelle aber ankommt, ist, dass diese Form der Resonanz nicht mit einer differenzierenden Empathie zu verwechseln ist ${ }^{54}$ und

51 Vgl. etwa N. Carroll: On some Affective Relations between Audiences and the Characters in Popular Fictions.

52 Ebd. S. 168.

53 Ebenso wenig ist es mit einem gemeinsamen Fühlen gleichzusetzen, bei dem das intentionale Gefühlsobjekt bewusst geteilt wird, wie das etwa bei einem komplexen Miteinander- oder geteilten Fühlen der Fall ist. Vgl. dazu die Konzeption von Angelika Krebs im Anschluss an Max Scheler in Krebs, Angelika: Zwischen Ich und Du. Eine dialogische Philosophie der Liebe, Berlin: Suhrkamp 2015, S. 220. Vgl. außerdem Carrolls Konzeption des »vectorially converging emotive state«, N. Carroll: On some Affective Relations between Audiences and the Characters in Popular Fictions, S. $171 \mathrm{ff}$.

54 Margrethe Bruun Vaage etwa unterscheidet deshalb zwischen einer bloßen unreflektierten Ansteckung und einer »embodied empathy«, bei der die Differenz zwischen einfühlendem und eingefühltem Subjekt klar ist. Diese »embodied empathy« Konzep- 
dass sie uns auch nicht hilft, a) zwischen den Gefühlen der Rezipientin und der Figur klar zu unterscheiden und b) die Hintergründe, Kontexte, Gründe bestimmter Gefühle zu erklären, denn dazu bedarf es der Imagination und der Reflexion. ${ }^{55}$ Wie Carroll schreibt, ist ihnen aber durchaus die Funktion zuzuschreiben, uns überhaupt für emotionale Reaktionen zu aktivieren, indem sie uns aufmerksam machen kann für die generelle Wertigkeit der Gefühle der Figuren. ${ }^{56}$

2. Bestimmte Vertreter einer Theory of Mind (ToM) sind dagegen der Ansicht, dass wir, um die Gefühls- und Interessenlagen anderer zu verstehen, eine allgemeine volkspsychologische Theorie anwendeten. Nach der sogenannten Theory Theory wenden wir als Rezipienten eine im Laufe der Kindheit erlernte basale Theorie des Geistes an (eine Art Volkspsychologie), mit der wir von bestimmten Mustern mentaler Zustände wie Emotionen, Stimmungen, Wünschen und Überzeugungen ausgehen und damit auch auf diese Zustände anderer inferentiell schließen, um deren Hintergründe und Handlungsfolgen zu verstehen. ${ }^{57}$ Nach der Theory Theory wird aus einer Dritten-Person-Perspektive allgemeines Wissen auf eine spezifische Situation eines anderen angewendet, das Verstehen einer anderen Person ist primär theoretischer Natur. ${ }^{58}$ Je weniger wir über eine fiktive Figur wissen, desto hilfreicher scheint eine solche Anwendung. Angenommen, wir werden Zeuge oben beschriebener Szene: So können wir, ohne etwas anderes zu wissen, schlussfolgern, dass der Junge, der hinter verschlossener

tion könnte daher gut zu dem passen, worauf ich weiter unten im Zusammenhang mit dem Ansatz von Edith Steins Phasentheorie der Empathie zu sprechen komme. Vgl. Vaage, Margrethe Bruun: »Fiction Film and the Varieties of Empathic Engagement«, in: Midwest Studies in Philosophy XXXIV (2010), S. 158-179, S. 163.

55 Vgl. zu Rolle der Reflexion auch N. Carroll: On some Affective Relations between Audiences and the Characters in Popular Fictions.

56 Ebd. S. 177 ff. Carroll spricht in diesem Zusammenhang von »Mirror Reflexes« und behandelt sie als eigene Kategorie im Unterschied zu den infektiösen Identifikationen. Wir können diese Kategorien aber meines Erachtens unter eine zusammenfassen, denn bei beiden liegt der Fall einer Gefühlsübereinstimmung auf leiblicher und präreflexiver Ebene vor.

57 Für einen hilfreichen, kritischen Überblick vgl. Zahavi, Dan/Overgaard, Søren: »Empathy without Isomorphism: A Phenomenological Account«, in: Jean Decety (Hg.), Empathy: From Bench to Bedside, Cambridge, MA: MIT Press 2012, S. 3-20.und Zahavi, Dan: Self and Other, Exploring Subjectivity, Empathy, and Shame, Oxford: Oxford University Press 2014.

58 S. Overgaard/D. Zahavi: Empathy without Isomorphism, S. 3; T. Breyer: Verkörperte Intersubjektivität und Empathie, S. 18 ff. 
Tür mit dem Rohrstock geschlagen wird, körperliche und seelische Schmerzen empfindet, dass er sich gedemütigt fühlt, dass sein Wille gebrochen wird und so weiter. Wir können auch einige Schlussfolgerungen auf die anderen Personen im Raum anstellen: Die Strenge des Pfarrers, die Angst der Geschwister am Esstisch etc. Wenn wir auch das kennen, was der Szene vorausliegt, können wir weitere Hintergründe narrativ ergänzen, unsere Theorie mit Wissen um die Figuren versorgen und über das Schlussfolgern dieses Wissen vervollständigen und auf dieser Grundlage Situationen wie die dargestellte dann verstehen. Wie aber kommen wir überhaupt zu einem solchen Wissen und welcher Art ist es?

Über das inferentielle Schließen kommen wir zwar zu einem propositionalem Wissen, einem »knowing that «: Wenn eine Figur in Tränen ausbricht, nachdem sie gerade gedemütigt wurde, so folgern wir, dass sie traurig, verletzt, verärgert ist und nicht, dass sie Freudentränen vergießt. Wir wissen allerdings nicht, wie es für sie ist, traurig, verletzt, verärgert zu sein, nachdem sie gedemütigt wurde. Dies ist einer der Schwachpunkte der Theory Theory: Sie ist schlicht zu theoretisch, um das, was andere Personen oder auch fiktive Figuren ausmacht, zu verstehen, nämlich ihre konkrete kontextuale und leibliche, verkörperte Eingebundenheit in eine Umwelt, innerhalb derer sie sich bewegen, interagieren und ausdrücken. Perspektiven sind konkrete Erfahrungsperspektiven, die uns über narrative Fiktionen in einer pointierten, prägnanten und verdichteten Weise vergegenwärtigt werden. ${ }^{59}$ Das, was die besondere epistemische und ethische Leistung von narrativer Fiktion im Besonderen und Kunst im Allgemeinen gegenüber anderen Wissensquellen wie etwa Sachbücher, Nachrichtenfilmen etc. auszeichnet, ist ihr non-propositionaler Erkenntniswert: Das Vermitteln eines erfahrungsbezogenen »knowing how«, des Wissens, wie sich etwas anfühlt oder wie es ist, in einer bestimmten Situation zu sein. ${ }^{60}$ Was genau in der Figur vorgeht und wie es sich für sie konkret anfühlt, bleibt uns bei dem rein kognitiv und vor allem theoretisch ablaufenden Schließen nach der Theory Theory verborgen. Nun ließe sich einwenden, dass uns dies auch bei fiktiven Figuren verborgen bleibt ${ }^{61}$, schon aus ontologischen Gründen, da diese fiktiven Figuren tatsächlich ja nicht fühlen,

59 Vgl. dazu etwa auch Gabriel, Gottfried: »Über Bedeutung in der Literatur. Zur Möglichkeit ästhetischer Erkenntnis«, in: Ders., Zwischen Logik und Literatur. Erkenntnisformen in Dichtung, Philosophie und Wissenschaft, Stuttgart: Metzler 1991, S. 2-18. u. I. Vendrell Ferran: Das Wissen der Literatur und die epistemische Kraft der Imagination.

60 Vgl. Gabriel, Gottfried: Erkenntnis, Berlin/Boston: De Gruyter 2015, S. 57 ff. u. S. 97 ff.

61 Diesen Hinweis verdanke ich Malte Hagener. 
sondern wir nur so tun, als ob sie fühlten. Ein anderer Grund, warum uns dies bei fiktiven Figuren verborgen bleiben könnte, ist, dass sie uns nicht wirklich leibhaftig gegenüberstehen. Die leibliche Interaktion des empathischen Prozesses wird hierbei - besonders beim Film - aber durch die expressiven Mittel ersetzt und kann durchaus ähnlichen phänomenalen Erfahrungscharakter haben. ${ }^{62}$

Um jemanden zu verstehen, reicht es nicht, Modelle auf andere anzuwenden. Auch ist mir nicht klar, wie dadurch Neues sichtbar werden kann. Das mag auch der Grund für das Argument von Jinhee Choi sein, wonach wir sogar umso mehr die Erfahrungsperspektive imaginieren müssten, je weniger wir wissen. ${ }^{63}$ Margrete Bruun Vaage nennt dies den $\gg$ restricted knowledge view on empathy «. ${ }^{64}$ Demnach würden wir gerade dann zum Imaginieren - und zwar aus der ErstenPerson-Perspektive - aufgefordert, wenn wir besonders wenig wissen. Ohne dass ich an dieser Stelle ausführlich auf dieses Argument eingehen kann, gibt es mindestens einen Schwachpunkt: Denn dies würde bedeuten, dass wir weniger mit Hauptfiguren perspektivisch imaginierten, sondern vor allem mit Nebenfiguren. Wir würden uns imaginativ viel mehr für diese engagieren, denn über diese wissen wir das wenigste. Das ist aber empirisch und handlungslogisch unplausibel. Außerdem führt das Imaginieren einer Perspektive, über deren Kontext, Hintergrund, Persönlichkeit etc. wir wenig bis gar nichts wissen, sehr schnell ins Leere oder ins Spekulieren.

3. Unter eine dritte Gruppe möchte ich die Simulationstheorien subsumieren, auch wenn es innerhalb dieser sehr heterogene Ansätze gibt. ${ }^{65}$ Die Simulationstheorie ist eine Gegenposition zur Theory Theory innerhalb der ToM-Debatte und behauptet, dass wir nicht theoretisches Wissen über den menschlichen Geist, sondern unsere eigenen mentalen Zustände als Modell verwenden, um mit Hilfe unseres Imaginationsvermögens die Zustände anderer zu erschließen. Wir stellen uns vor bzw. simulieren, wie wir in der Situation des anderen fühlen würden. ${ }^{66}$ Goldman argumentiert, dass unser Verstehen darin gegründet sei, dass wir uns

62 Vgl. dazu auch das Konzept des Leihkörpers von Christiane Voss im Anschluss an Sobchack, C. Voss: Der Leihkörper. Erkenntnis und Ästhetik der Illusion.

63 Choi 2005, 23, zitiert nach M. Bruun Vaage: Fiction Film and the Varieties of Empathic Engagement.

64 M. Bruun Vaage: Fiction Film and the Varieties of Empathic Engagement, S. 162.

65 Vgl. Goldman 2006; K.R. Stueber: Rediscovering Empathy, Agency, Folk Psychology, and the Human Sciences.

66 Vgl. T. Breyer: Verkörperte Intersubjektivität und Empathie, S. 18 ff. 
selbst imaginativ in die Situation des Anderen hineinprojizieren würden. ${ }^{67}$ Peter Goldie nennt dies »In-his-shoes-Imagining«: man nimmt die eigenen Eigenschaften und Hintergründe mit in die imaginierte Situation. ${ }^{68}$ Am Beispiel der oben beschriebenen Filmszene illustriert, heißt das, dass wir uns vorstellen, wie wir an der Stelle des Jungen fühlen würden, wenn wir in dem Esszimmer von unserem Vater mit dem Rohrstock verprügelt würden.

Für diese Sichtweise gibt es wiederum Pro- und Contra-Argumente: Ein Vorteil ist, dass uns die Konzeption hilft zu erklären, wie wir neue Perspektiven mit unseren eigenen in Verbindung bringen. Um darüber zu reflektieren, wie wir das Fühlen und Handeln des Anderen beurteilen, kann es hilfreich sein, zu imaginieren, wie wir selbst in derselben Situation fühlen und handeln würden. Ein zweiter Vorteil ist, dass diese Konzeption dem Zuschauer wesentlich mehr Aktivität und kognitive Eigenleistung zugesteht als zum Beispiel die passiven Ansteckungs- und Spiegeltheorien. Allerdings, und dies sind die ersten beiden problematischen Punkte, gibt es einen epistemischen und einen normativen Einwand: Der erste Einwand lautet, dass wir, wenn wir simulieren sollen, in der Situation des Jungen zu sein, uns konsequenterweise auch vorstellen sollten, unser, nicht sein Vater würde uns schlagen. Denn was haben wir bzw. hat unsere persönliche Perspektive schließlich mit seinem Vater zu tun? Doch dann bewegen wir uns sehr weit von der eigentlichen Perspektive, die wir verstehen wollen, weg, nämlich der des Jungen, der am Vorabend des Ersten Weltkriegs von einem sehr strengen Vater, der Pfarrer ist, gezüchtigt wird. Oder stellen wir uns vor, wir würden eben zu jener historischen Zeit von einem sehr strengen Vater geschlagen werden, der Pfarrer ist? Auch das ist eher unplausibel. Ungeachtet der epistemischen Schwierigkeiten, würde ein normativer Einwand lauten, dass durch die egozentrische Simulation nicht die konkrete Fremdperspektive eingenommen wird, also nichts Neues kennen gelernt wird, womit wir unsere eigene Perspektive reflektieren könnten. Die Simulationstheorie bleibt einem cartesianischen Denken verhaftet, wonach uns nur unsere eigenen mentalen Zustände zugänglich seien. Dann aber handelt sich bei dem »In-his-shoes-Imagining « nur um das Spiel mit der eigenen Perspektive und ist somit eine »egozentrische. Transposition «. ${ }^{69}$ Dies ist von einer allozentrischen Perspektiveneinnahme zu unterscheiden, bei der die komplexe Sichtweise, Persönlichkeit und Perspektive des Anderen

67 Vgl. zur Darstellung dieser und der anderen Positionen D. Zahavi: Self and Other, Exploring Subjectivity, Empathy, and Shame.

68 Vgl. Goldie, Peter: The Emotions. A Philosophical Exploration, Oxford: Clarendon Press 2000.

69 T. Breyer: Verkörperte Intersubjektivität und Empathie, S. 169. 
berücksichtigt werden soll. ${ }^{70}$ Es gibt aber eine Variante innerhalb der Simulationstheorie, welche von der Möglichkeit eines »central imagining « oder »imagining from the inside « ausgeht und den Cartesianismus aufhebt. ${ }^{71}$ Demnach vollzögen wir aus der Perspektive des Anderen Überzeugungen und Gefühle nach und kämen über eine Form des »reenactments « zu übereinstimmenden Gefühlen. ${ }^{72}$ Der technisch ideale Auslöser dafür kann der Point of View Shot sein, also die subjektive Kameraperspektive. Ein Problem bei diesem Ansatz ist jedoch, dass er davon ausgeht, dass es zu einer affektiven Identifikation zwischen einfühlendem und eingefühltem Subjekt komme. Aber muss ich tatsächlich genau dasselbe fühlen, um zu verstehen, wie es dem Anderen geht ${ }^{73}$ Beziehungsweise, ist eine Gefühlsübereinstimmung nicht eher eine neue affektive Phase, ein sympathisches Mitfühlen? Ein weiteres Problem der Simulationstheorien - und dies betrifft beide Varianten - ist, dass die Konzeptionalisierung dieses Vorgangs wiederum vor allem kognitivistischer Natur ist; die expressiven Eigenschaften des Films und der dargestellten Situation und damit auch die leibliche Adressierung und Involvierung des Filmzuschauers finden hier zu wenig Beachtung. In den vergangenen Jahren sind phänomenologische Ansätze und Theorien der Verkörperung zunehmend in den Vordergrund gerückt, die die Rolle des Leiblichen, der Sinne und des Expressiven stärker berücksichtigen. ${ }^{74}$

4. Ich möchte mich im Folgenden für eine phänomenologisch orientierte Empathie-Konzeption aussprechen: Phänomenologische Ansätze rücken die unmittelbare Wahrnehmung expressiver Qualitäten in den Vordergrund. ${ }^{75}$ In meiner Erweiterung und Anwendung dieses Ansatzes auf den fiktionalen Film kommt innerhalb des empathischen Prozesses aber noch ein imaginativer Vor-

70 Ebd. S. $27 \mathrm{f}$.

71 Vgl. z.B. K.R. Stueber: Rediscovering Empathy, Agency, Folk Psychology, and the Human Sciences.

72 Ebd.

73 Vgl. für eine Kritik dazu P. Goldie: The Emotions. A Philosophical Exploration.

74 Vgl. zu dieser Entwicklung die spannende Filmtheorie zur Einführung von Thomas Elsaesser und Malte Hagener, die sich in ihrer Diskussion der verschiedenen Theorien stets an der jeweiligen Beziehung zwischen Film und Körper orientiert. Vgl. Elsaesser, Thomas/Hagener, Malte: Filmtheorie zur Einführung, Hamburg: Junius 2007, zum Trend der Verkörperungstheorien insb. S. 212 ff. Vgl. außerdem J. Fingerhut et al.: Philosophie der Verkörperung. Grundlagentexte zu einer aktuellen Debatte.

75 Vgl. etwa Zahavis »direct perception proposal«: Zahavi, Dan: »Empathy and Direct Social Perception. A Phenomenological Proposal «, in: Review of Philosophy and Psychology 2 (2011), S. 541-558. 
gang zur direkten Wahrnehmung hinzu. Über die imaginative Perspektiveneinnahme können wir erst, so meine These, den komplexen Gründen und Kontexten der Gefühlsexpression auf den Grund gehen. Da es hierbei darum geht, sich Gefühle und damit zusammenhängende mentale Prozesse zu vergegenwärtigen, ohne dass sie notwendig gegenwärtig (aktual) werden, nenne ich meinen Ansatz »Vergegenwärtigungsansatz «. ${ }^{76}$

Zur Empathie gehört nach diesem Ansatz ein Prozess, bei dem die spezifische Lage einer Person, ihre Gefühle und Handlungen wahrgenommen werden, und zwar wie sie sich in der leiblichen Expression und aus ihrer spezifischen Perspektive manifestieren. Die Perspektiveneinnahme wäre dann eine, welche nicht nur kognitiv, sondern auch leiblich abläuft, insofern es immer auch Perspektiven von Körpern, Dingen, Stimmungen sind, die vergegenwärtigt werden. Vor allem aber kommt die Imagination noch hinzu, um Kontexte und Gründe einer Gefühlssituation nachvollziehen zu können.

Anhand mehrerer Textstellen in Edith Steins Dissertation »Das Problem der Einfühlung « lässt sich Empathie als ein Prozess bestehend aus drei auf einander aufbauenden Phasen plausibilisieren. ${ }^{77}$ Die erste Phase besteht in der Wahrnehmung des unmittelbaren Ausdrucks: Der Ausdruck des Anderen taucht »mit einem Schlage « vor uns auf. ${ }^{78}$ Der andere wird als Anderer (an)erkannt. Dies ist verbunden mit einer direkten, non-inferentiellen Wahrnehmung der Emotionen, Sinneswahrnehmung oder anderen mentalen Zuständen des Anderen, die anhand des Ausdrucks unmittelbar verstanden werden. ${ }^{79}$ Im Unterschied zur Position von Lipps mündet dieser basale Vorgang aber nicht in einer Ansteckung oder Nachahmung des fremden Gefühls. Es ist daher auch nicht mit einer »mimetischen Resonanz « zu verwechseln. ${ }^{80}$ Es ist eine Wahrnehmung auf ganzheitlicher Ebene und darum schon relativ informativ: Perzeption ist nicht nur visuell, wir nehmen unsere Umwelt - und daher auch das Geschehen auf der Leinwand - mit unserem ganzen eingebetteten Leib wahr und nehmen den Ausdrucksleib des

76 Edith Stein verwendet in ihrer Dissertation zur »Einfühlung« selbst den Ausdruck »Vergegenwärtigung«, vgl. Stein, Edith: Zum Problem der Einfühlung, Freiburg im Breisgau: Herder 2008 (1917), insb. S. 16, S. 20, S. 23. Vgl. zu diesen Positionen außerdem etwa S. Overgaard/D. Zahavi: Empathy without Isomorphism; T. Breyer: Verkörperte Intersubjektivität und Empathie, insb. S. 208.

77 Man könnte auch von Stufen, Vollzugsebenen oder Etappen sprechen.

78 E. Stein: Zum Problem der Einfühlung, S. 19.

79 S. Overgaard/D. Zahavi, Empathy without Isomorphism, S. 16.

80 T. Breyer: Verkörperte Intersubjektivität und Empathie, S. 51. 
Anderen wahr, der Bedeutungsträger ist für die gesamte Situation. ${ }^{81}$ Beim Film wird dies durch die Körper und Dinge, durch die Atmosphäre, die ästhetischen Mittel etc. unterstützt: In der beschriebenen Szene aus DAS WEISSE BAND nehmen wir die Spannung der gesamten Szene zum Beispiel über das Mittel der einzigen Einstellung und die fließende Bewegung der Kamera wahr - es gibt keinen Schnitt, keine Unterbrechung. Auch dass eine Stille in einer klar abgesteckten Struktur herrscht, wird leiblich wahrgenommen. Vor allem aber sind es die Schreie des Jungen, die wir hören, während unsere Augen starr den langen Flur entlang blicken, die »mit einem Schlage« vor uns auftauchen. Nun haben wir es aber im Fall des fiktionalen, narrativen Films mit einem entscheidenden Unterschied zur Wirklichkeit zu tun: Während phänomenologische Ansätze davon ausgehen, dass es bei der intersubjektiven Empathie auf das direkte Aufeinandertreffen zweier lebendiger Körper ankommt, trifft der Zuschauerkörper beim Film auf einen zweidimensionalen Leinwandkörper, auf den Körper von Schauspielern projiziert sind, die fiktionale Figuren repräsentieren. Eine echte Interaktion kann aus filmontologischen Gründen nicht stattfinden. ${ }^{82}$ Stattdessen können aber die expressiven Mittel für einen Eindruck des Lebendigseins und der Adressie-

81 Christiane Voss entwickelt dazu im Anschluss an Vivian Sobchacks Konzept des »cinesthetic subject« die Idee des »Leihkörpers«, der sich zwischen Leinwand und realem Zuschauerkörper schiebt und der das Zweidimensionale der Leinwand in Dreidimensionalität verwandelt und ihr damit Körperlichkeit - und ich würde mit Blick auf meine oben angegebenen Bedeutungen von »Perspektive - verkörperte Perspektive verleiht. Mit Blick auf die Konzeption Steins ließe sich der empathische Vorgang dann als einer beschreiben, bei dem dieser perspektivische Leihkörper imaginativ betreten wird bzw. dieser vom Zuschauer als dreidimensionaler Körper den Figuren »geliehen« wird. Voss schreibt hier von einer sensorisch-affektiven und geistigen Resonanz, weshalb ihre Konzeption, anders als die stark narratologisch- und hermeneutischkritische Position Sobchacks, mit der hier von mir vorgestellten Konzeption von Empathie als ganzheitlicher, komplexer Prozess aus Körperausdruck, -wahrnehmung und imaginativer Vergegenwärtigung vereinbar ist. Vgl. C. Voss: Der Leihkörper. Erkenntnis und Ästhetik der Illusion, insb. S. 108ff. Sobchack sieht die eigentlichen Verstehensleistungen in der filmästhetischen Erfahrung gerade nicht in Reflexionsbemühungen, sondern in den unmittelbaren, präreflexiven leiblichen Reaktionen manifestiert. Vgl. dazu C. Voss: Der Leihkörper. Erkenntnis und Ästhetik der Illusion, S. 115.

82 Breyer spricht mit Verweis auf Fuchs von »fiktionaler Empathie«, T. Breyer: Verkörperte Intersubjektivität und Empathie, S. 218 f. 
rung sorgen. ${ }^{83} \mathrm{Zu}$ diesem Eindruck gehören neben der Illusion - des Als-ObBewusstseins - eine Form der Imagination: Imagination wird häufig reduziert auf den mentalen Vorgang, sich eine Situation eines Anderen bildlich zu repräsentieren. Imagination ist aber viel komplexer und meint unter anderem auch, etwas »als etwas « zu sehen, zum Beispiel die Schauspieler als fiktionale Charaktere einer Geschichte wahrzunehmen (und nicht bloß als Schauspieler). Wir setzen auch unsere Imagination ein, um in jemandes Ausdruck etwas zu sehen, zum Beispiel seine Traurigkeit. Imagination vollbringt eine Syntheseleistung, die für die Deutung von etwas notwendig ist. So ergänzen wir auch das Filmbild um das, was man nicht im Bild sieht, was aber zur Situation dazu gehört. ${ }^{84}$

Zur näheren Erläuterung möchte ich abermals auf die beschriebene Szene zurückkommen: Der direkten Perzeption sind hier nämlich Grenzen gesetzt. Der Leib des Jungen ist nur durch seine Schreie gegenwärtig. Dadurch ist er uns nur eingeschränkt zugänglich. Gleichzeitig sind wir visuell auf die Ausdrucksqualität der Kameraperspektive gerichtet. Bereits wie im ersten Filmbild zu Beginn des Films sind wir synchron auf zwei Perspektiven eingelassen, eine visuelle und eine auditive, die wir mit Hilfe unserer Imagination verbinden. Zweitens ist das Ganze eingebettet in einen narrativen Kontext, den wir imaginativ ergänzen. Beide Aspekte weisen auf eine Distanz und Handlungsunfähigkeit des Zuschauers hin, der betrachten, aber nicht aktiv handeln - zum Beispiel zur Tür gehen kann. Zudem ließe sich angesichts dieser Distanz herstellenden Ästhetik auch die Frage aufwerfen, ob wir an dieser Stelle überhaupt nur Emotionen gegenüber dem narrativen Geschehen entwickeln oder eher auf eine Metabene mit ArtefaktEmotionen, wie Ed Tan sie konzipiert hat, gehoben werden. ${ }^{85}$ Eine Artefakt Emotion richtet sich auf den Film als ästhetisches Artefakt und nicht auf die Narration. In jedem Fall scheint die direkte Wahrnehmung in dieser Szene für das Erfassen und Vergegenwärtigen der eigentlich relevanten Gefühlssituation nicht ausreichend zu sein, um sich ein umfassendes Bild der Perspektive der Situation zu vergegenwärtigen. Aber wir können durchaus auf diesem Wege die Metaperspektive des impliziten Autors, Regisseurs oder Films als Ganzem erfahren, der

83 Vgl. abermals das Konzept des Leihkörpers von C. Voss: Der Leihkörper. Erkenntnis und Ästhetik der Illusion sowie V. Sobchack: The Address of the Eye. A Phenomenology of Film Experience.

$84 \mathrm{Zu}$ den verschiedenen Bedeutungen von Imagination vgl. die sehr ausführliche und analytisch sehr saubere Darstellung von R. Scruton: Art and Imagination. A Study in the Philosophy of Mind.

85 Tan, Ed: Emotion and the Structure of Narrative Film: Film as an Emotion Machine, Mahwah, NJ: Erlbaum 1996, S. 81 ff. 
über solche Mittel das Gemachtsein der Geschichte und die damit verbundene Hilflosigkeit der Zuschauer aufdeckt.

Um sich nun die konkreten Gefühle des Jungen und deren Gründe erfahrbar und erklärbar zu machen, muss zum leiblich-perzeptiven ein mentaler, imaginativer Vorgang der Vergegenwärtigung der Situation hinzukommen, der auch das gesamte Narrativ, das heißt, das was vor der Situation liegt und was sie antizipiert, berücksichtigt. Dies ist ein komplexer kognitiver Vorgang, der meines Erachtens zu einem höherstufigen Prozess der Empathie dazugehört; auch beschreibt Stein den Vorgang so, dass es nach der direkten Wahrnehmung des Ausdrucksverhaltens zu einem Hineinversetzen kommt: In der zweiten Phase oder »Vollzugmodalität«, wie Stein schreibt, tritt man dann an die Stelle des Anderen, wendet sich »seinem Objekt« zu, das heißt, seinem Gefühlsobjekt. Stein bezeichnet diesen Vorgang als »erfüllende Explikation «. ${ }^{86}$ Aufbauend auf dem, was wir bereits leiblich unmittelbar erfahren haben, können wir uns nun die ganzheitliche Erfahrungsperspektive der Person bzw. fiktiven Figur imaginativ vergegenwärtigen. »Erfahrung« impliziert, wie zuvor unter Rückgriff auf den anspruchsvollen Begriff Deweys angedeutet, Leiblichkeit, Kontextualität, Situierung, Historizität, Narrativität sowie Denken, Fühlen, Handeln, welche sich in den je konkreten Perspektiven entfalten; die Gesamtheit der Erfahrungsperspektive kann aber nicht selbst nur leiblich erfasst, sondern muss auch imaginativ vergegenwärtigt werden. Auf dieser zweiten Vollzugsebene kann es nach Stein zwar zu einem »inneren Mitmachen« der eingefühlten »Tendenzen«kommen, das aber wiederum nicht $\mathrm{zu}$ verwechseln sei mit einem identischen »Einssein « oder mit einem »originären Erleben $\ll{ }^{87}$ Der Vorgang ist stets mit einer Ich-DuDifferenz markiert und geht mit dem Bewusstsein der einfühlenden Person einher, dass sie nicht selbst die fühlende Instanz ist. Anders als bei einer mimetischen Resonanz der präreflexiven Gefühlsadaption, wie ich sie am Beispiel Lipps beschrieben habe, führt dieser Vorgang gerade nicht zu einem Verlust des Selbst- und Weltgewahrseins. Mehr noch: Die Perspektiven des Anderen werden vor ihrem jeweiligen Hintergrund, aber auch vor dem Hintergrund der je eigenen Perspektiven der empathischen Person vergegenwärtigt, gerade weil wir als Personen die Welt immer aus einer bestimmten Perspektive wahrnehmen, wie Merleau-Ponty betont: »Heißt Sehen nicht immer, irgendwoher sehen? «. ${ }^{88}$ Anders aber als beim theoretischen Nachvollzug ist dies auch nicht nur ein rein kogniti-

86 E. Stein: Zum Problem der Einfühlung, S. 19.

87 Ebd. S. 22.

88 Merleau-Ponty, Maurice: Phänomenologie der Wahrnehmung, Berlin: De Gruyter 1966, S. 91. 
ver Vorgang und ein »Wissen, dass«, sondern vielmehr ein Erfahrungswissen, ein »Wissen, wie«, denn wir vergegenwärtigen uns die Situation leibhaftigexpressiv und lebhaft-imaginativ. Auch anders als bei der Simulationstheorie ist dies nicht bloß ein kognitiver Vorgang, bei dem die Geschehnisse mental repräsentiert werden oder bei dem wir uns nur selbst in der gegebenen Situation vorstellen, sondern es geht um die Perspektive des Anderen. Gleichzeitig kommt es aber nicht notwendig zu einer Gefühlsreplikation, sondern qua direkter leiblicher Wahrnehmung steigen wir in die Situation ein und vergegenwärtigen sie uns konkret, indem wir uns an die Stelle des Anderen versetzen, ohne aber das Gleiche fühlen zu müssen; ein originäres Gefühl wäre vielmehr eine neue Modalität: Ein paralleles Fühlen oder sympathisches Mitfühlen. Es gibt dazu eine interessante, aber nicht gerade sehr einfach zu deutende Textstelle bei Max Scheler, der schreibt, dass es sich bei dem empathischen Vorgang (er nennt ihn »Nachfühlen« oder »Nachleben«) wohl um »ein Fühlen des fremden Gefühls« handele. Es sei »kein bloßes Wissen um es oder nur ein Urteil, der Andere habe das Gefühl; gleichwohl ist es kein Erleben des wirklichen Gefühls als eines Zustandes. Wir erfassen im Nachfühlen noch die Qualität des fremden Gefühls - ohne daß es in uns herüberwandert oder ein gleiches reales Gefühl in uns erzeugt wird. ${ }^{89}$ Das Erfassen der Qualität ließe sich mit dem »inneren Mitmachen«, wie Stein es beschreibt, vergleichen, das stattfindet, ohne dass es zu einem originären Gefühl kommt: Meine Erachtens kommt es hier zu einem Nachfühlen der Gefühlsqualität, aber nicht zu einem Übernehmen des vollständigen Gefühls mit seiner Intentionalität, Evaluierung und Kontextualität. Gerade aber weil die erste Phase eine des Wahrnehmens des leiblichen Ausdrucks ist und gerade weil uns die Expressivität unmittelbar leiblich berührt, ist hier nach meiner Lesart davon auszugehen, dass der phänomenale Grundton des Gefühls als gut oder schlecht, schwer oder leicht, bedrohlich oder entspannend, lustvoll oder unlustvoll etc. eine Resonanz hervorruft, also auf einer leiblichen Ebene gespürt wird, denn gerade diese ist nicht allein dem Subjekt gegenwärtig, sondern befindet sich auch als Atmosphäre im »intersubjektiven Raum $\ll .{ }^{90}$ Oder, um es am spezifischen Beispiel der filmästhetischen Erfahrung zu modifizieren, in dem über die expressiven Mittel des Films entstehenden Resonanzraum zwischen Zuschauerkörper und Filmkör-

89 Scheler, Max: Wesen und Formen der Sympathie, Frankfurt am Main: SchulteBulmke 1948 (Herv. i.O.).

90 Slaby, Jan: »Möglichkeitsraum und Möglichkeitssinn. Bausteine einer phänomenologischen Gefühlstheorie«, in: Kerstin Andermann/Undine Eberlein, Gefühle als Atmosphären. Neue Phänomenologie und philosophische Emotionstheorie, Berlin: Akademie Verlag 2011, S. 125-138, hier S. 133. 
per. Deshalb ist selbst eine solche komplexe Empathiekonzeption, welche mit imaginativen Vorgängen wie der Perspektiveneinnahme einhergeht, mit einem Konzept von leiblicher Resonanz zu vereinen. Gerade über das Zusammenspiel von leiblichem Mitschwingen und imaginativer Vergegenwärtigung können außerdem zusätzliche Perspektiven oder Aspekte einer Gefühlssituation aufgefangen werden: Denn Emotionen treten nicht einzeln auf, noch sind sie bloß ein punktuelles Ereignis. Sie sind vielmehr als Teil eines Clusters aus anderen Emotionen, Stimmungen und Hintergrundgefühlen zu verstehen, stehen im Kontext bestimmter Wertmaßstäbe, Überzeugungen oder Konventionen und haben damit auch eine sehr komplexe narrative und persönliche Struktur. Die dritte Phase nach dem Stein'schen Modell enthält schließlich eine »Klärung «, »Vergegenständlichung « oder »Reflexion « des fremden Erlebnisses. ${ }^{91}$ Hier tritt die empathisierende Person quasi wieder einen Schritt zurück in die distanzierte Beobachterposition, worin sich dieser Vorgang zum Beispiel von einem Ansteckungsvorgang unterscheidet.

Indem der empathische Prozess aus drei Phasen besteht, kann auch er selbst als ein narrativer Erfahrungsvorgang bezeichnet werden, der einen Anfang, nämlich das visuelle und leibliche Wahrnehmen des Ausdrucks, eine Mitte, nämlich den Perspektivenwechsel, und einen Abschluss, nämlich die reflektierende $\mathrm{Ob}$ jektivierung aufweist. Da dieser Prozess leiblich und geistig so komplex ist, produziert er zwar nicht notwendig sichereres oder detaillierteres Wissen über den Zustand des Anderen als etwa die anderen möglichen Formen des »mindreading «, wie sie etwa die Theory Theory oder die Simulationstheorien vorschlagen. Er ist aber auf den Anderen in seiner Ganzheitlichkeit bezogen und nimmt diesen als Anderen ernst, versucht also den Anderen als konkreten Anderen mit seiner partikularen Perspektive zu verstehen..$^{92}$ Der Empathie ist in dieser Form denn auch eine ethische »Einstellung eingeschrieben, die die Fremdheit als solche registriert, respektiert und in den Imaginationsprozess integriert. « ${ }^{93} \mathrm{Ob}$ man richtig liegt, ist dann Sache der Interpretation, vor allem bei Kunst und Fiktionen, welche sich einer eindeutigen propositionalen Festlegung ja gerade entziehen. Oder, wie zuvor angegeben, ist vielmehr von einem »Wissen« in einem weiteren, nämlich nicht-propositionalen »Wissen, wie es ist« auszugehen, wel-

91 E. Stein: Zum Problem der Einfühlung, S. 17, S. 19 u. S. 51.

$92 \mathrm{Zu}$ Empathie als ein »ernst nehmen« vgl. auch A. Neill: Empathie und (filmische) Fiktion, in diesem Band.

93 T. Breyer: Verkörperte Intersubjektivität und Empathie, S. 207. Zur Empathie als »Wissen« vgl. Schmalzried in diesem Band. 
ches als nicht-propositionales nicht auf einen Begriff oder eine wahrheitsfähige Aussage zu reduzieren ist.

\section{FAZIT}

Ich habe zwischen verschiedenen Arten von Perspektiven unterschieden und verschiedene Theorieansätze der empathischen Resonanz vorgestellt, die zum Einsatz kommen können, wenn ZuschauerInnen auf die Perspektivenangebote von fiktiven narrativen Filmen reagieren. Dabei habe ich selbst einen imaginativleiblichen Vergegenwärtigungsansatz entwickelt, der meines Erachtens das trifft, wie wir auf die komplexen Perspektiven von fiktionalem Film und seinen Figuren sowie deren Zusammenspiel auf narrativer wie expressiver Ebene umfassend reagieren oder reagieren sollten, um die konkreten und komplexen Perspektiven zu verstehen. Dabei habe ich im Anschluss an Edith Stein gezeigt, dass wir uns die Gefühle, Stimmungen und Atmosphären, wie sie der Film und seine fiktiven Figuren zum Ausdruck bringen, in drei aufeinanderfolgenden und miteinander in dynamischer Wechselwirkung stehenden Phasen vergegenwärtigen: Eine direkte sensorisch-leibliche Ausdruckswahrnehmung, ein explizierendes Sich-Hineinversetzen und eine reflektierende Objektivierung. Dieser Prozess ist nicht notwendig mit einer Gefühlsübertragung verbunden. Vielmehr kann es zu einem Mitschwingen kommen, die Entwicklung eines vollen Gefühls wäre aber ein neues Phänomen.

Aufgrund der Komplexität einer solchen Empathie ließe sich einwenden (wie etwa Goldie in Bezug auf das »imaginative perspective-shifting «), dass diese eher selten, und wenn nur in einfach strukturierten Fällen gelingt. ${ }^{94}$ Im Bezug auf narrative Fiktionen würde ich jedoch behaupten, es ist genau das Gegenteil: Wie oben bereits angegeben, erzählen narrative Fiktionen detailreiche Geschichten und lenken die Aufmerksamkeit ihrer RezipientInnen auf bestimmte, verdichtete Aspekte: Sie sind vorperspektiviert und affektiv präfokussiert. Fiktive narrative Filme, Literatur sowie die neuen Langzeitserien des so genannten »Quality TV « eignen sich für die Erläuterung und Erfahrung der komplexen Vorgänge der vergegenwärtigenden Empathie darum besonders gut, da sie in einem mehr oder weniger abgeschlossenen Rahmen eine Geschichte einer oder mehrerer Figuren kompakt und ausführlich erzählen. Das heißt aber auch, sie statten die RezipientInnen mit einem handlungslogischen und figurenbezogenen Wissen aus, wie es im alltäglichen Leben bei Begegnungen mit anderen Personen üblicherweise

94 P. Goldie: The Emotions. A Philosophical Exploration, S. 194 ff., S. 203. 
nicht so gegeben ist. Selbst wenn Gefühle und Handlungen der Figuren ambivalent sind, müssen sie nicht opak sein, sondern bieten der Rezeption vielmehr ein vielseitiges Perspektivenangebot, sie zu interpretieren. Der Film im Besonderen eignet sich darüber hinaus für die hier entwickelte Form der Empathie in einer Weise, da er aufgrund seiner hohen Expressivität auch die leibliche Seite des Prozesses sehr stark anspricht und so auch ein spezifisches Wissen des Körpers involviert.

\section{LITERATUR}

Assheuer, Thomas/Haneke, Michael: Nahaufnahme Michael Haneke. Gespräche mit Thomas Assheuer, Berlin/Köln: Alexander Verlag 2008.

Aumont, Jacques: »Der Point of View«, in: montage/av 16/1 (2007), S. 13-44.

Böhme, Gernot: Atmosphäre: Essays zur neuen Ästhetik, Frankfurt: Suhrkamp 2013.

Branigan, Edward: Narrative Comprehension and Film. London, New York: Routledge 1992.

Breyer, Thiemo: Verkörperte Intersubjektivität und Empathie, Frankfurt am Main: Vittorio Klostermann 2015.

Carroll, Noël: »Film, Emotion, and Genre«, in: Carl Plantinga/Greg M. Smith (Hg.), Passionate Views, Baltimore, MD: Johns Hopkins University Press 1999, S. 21-47.

Carroll, Noël: »On some Affective Relations between Audiences and the Characters in Popular Fictions«, in: Amy Coplan/Peter Goldie (Hg.), Empathy. Philosophical and Psychological Perspectives, Oxford: Oxford University Press 2011, S. 162-184.

Cavell, Stanley: »Was wird aus den Dingen im Film?«, in: Dimitri Liebsch (Hg.), Philosophie des Films. Grundlagentexte, Paderborn: Mentis 2006, S. 100-110.

Ders.: The World Viewed. Reflections on the Ontology of Film. Enlarged Edition, Cambridge, MA: Harvard University Press 1979.

Cieutat, Michel/ Rouyer, Philippe: Haneke über Haneke, Berlin/Köln: Alexander Verlag 2013.

Coplan, Amy/Goldie, Peter (Hg.): Empathy. Philosophical and Psychological Perspectives, Oxford: Oxford University Press 2011.

Curtis, Robin: »Immersion und Einfühlung«, in: montage/av,17/2 (2008), S. 89107. 
Deines, Stefan/Liptow, Jasper/Seel, Martin: »Kunst und Erfahrung. Eine theoretische Landkarte«, in Dies. (Hg.), Kunst und Erfahrung. Beiträge zu einer philosophischen Kontroverse, Berlin: Suhrkamp 2013, S. 7-37.

Dewey, John: Art as Experience, Carbondale, IL: Southern Illinois University Press 1989. [erstmals 1934]

Elsaesser, Thomas/Hagener, Malte: Filmtheorie zur Einführung, Hamburg: Junius 2007.

Fingerhut, Joerg/Hufendiek, Rebekka/Wild, Markus (Hg.): Philosophie der Verkörperung. Grundlagentexte zu einer aktuellen Debatte, Berlin: Suhrkamp 2013.

Fuchs, Thomas: »Zwischen Leib und Körper«, in: Martin Hähnel, Marcus Knaup (Hg.), Leib und Leben. Perspektiven für eine neue Kultur der Körperlichkeit, Darmstadt: Wissenschaftliche Buchgesellschaft 2013, S. 82-93.

Gabriel, Gottfried: Erkenntnis, Berlin/Boston: De Gruyter 2015.

Ders... »Fiktion, Wahrheit und Erkenntnis der Literatur«, in: Christoph Demmerling/Ingrid Vendrell Ferran (Hg.), Wahrheit, Wissen und Erkenntnis in der Literatur, Berlin: Akademie-Verlag 2014, S. 163-180.

Ders.: »Über Bedeutung in der Literatur. Zur Möglichkeit ästhetischer Erkenntnis«, in: Ders., Zwischen Logik und Literatur. Erkenntnisformen in Dichtung, Philosophie und Wissenschaft, Stuttgart: Metzler 1991, S. 2-18.

Genette, Gérard: Die Erzählung, München: Fink 1998.

Goldie, Peter: The Emotions. A Philosophical Exploration, Oxford, New York: Clarendon Press 2000.

Goldman, Alvin Ira: Simulating Minds: The Philosophy, Psychology, and Neuroscience of Mindreading, New York: Oxford University Press 2006.

Haneke, Michael: Das weiße Band. Eine deutsche Kindergeschichte. Das Drehbuch zum Film, Berlin: Berlin Verlag 2010.

Iser, Wolfgang: Das Fiktive und das Imaginäre, Frankfurt am Main: Suhrkamp 1991.

Koch, Gertrud: Breaking Bad, Zürich/Berlin: Diaphanes 2015.

Dies. (Hg.): Perspektive - Die Spaltung der Standpunkte. Zur Perspektive in Philosophie, Kunst und Recht, München: Fink 2010.

Krebs, Angelika: Zwischen Ich und Du. Eine dialogische Philosophie der Liebe, Berlin: Suhrkamp 2015.

Lipps, Theodor: Leitfaden der Psychologie, Leipzig: Wilhelm Engelmann 1903.

Merleau-Ponty, Maurice: Phänomenologie der Wahrnehmung, Berlin: De Gruyter 1966.

Misselhorn, Catrin: »Literatur, Wahrheit und Philosophie«, in: Catrin Misselhorn/Schamma Schahadat/Irina Wutsdorff (Hg.), Erkenntnis und Darstel- 
lung: Formen der Philosophie und der Literatur, Paderborn: Mentis 2011, S. 21-39.

Dies.: »Ästhetische Erfahrung und die Perspektive der ersten Person«, in: Thomas Grundmann et al. (Hg.), Anatomie der Subjektivität. Bewusstsein, Selbstbewusstsein und Selbstgefühl, Frankfurt am Main: Suhrkamp 2005, S. 417-437.

Neill, Alex: »Empathy and (Film) Fiction«, in: Noël Carroll/David Bordwell (Hg.), Post-Theory. Reconstructing Film Studies, Wisconsin: University of Wisconsin Press 1996, S. 175-194.

Nünning, Ansgar: »Die Funktion von Erzählinstanzen. Analysekategorien und Modelle zur Beschreibung des Erzählerverhaltens«, in: Literatur in Wissenschaft und Unterricht 30 (1997), S. 323-349.

Nussbaum, Martha: Upheavals of Thought. The Intelligence of Emotions, Cambridge: Cambridge University Press 2003.

Rizzolatti, Giacomo/Fadiga, Luciano/Fogassi, Leonardo/Gallese, Vittorio: »Resonance Behaviour and Mirror Neurons«, in: Archives Italiennes de Biologie Nr. 137 (1999), S. 85-100.

Rorty, Richard: »Der Roman als Mittel zur Erlösung aus der Selbstbezogenheit«, in: Joachim Küpper/Christoph Menke (Hg.), Dimensionen ästhetischer Erfahrung, Frankfurt am Main: Suhrkamp 2003, S. 49-66.

Rosa, Hartmut: Resonanz. Eine Soziologie der Weltbeziehung, Berlin: Suhrkamp 2016.

Scheler, Max: Wesen und Formen der Sympathie, Frankfurt am Main: SchulteBulmke 1948. [erstmals 1923]

Schmitz, Hermann: Der Leib, der Raum und die Gefühle, Bielefeld: Edition Sirius 2007.

Schulte-Sasse, Jochen: »Perspektive/Perspektivismus« (= Ästhetische Grundbegriffe, Band 4), Stuttgart/Weimar: Metzler 2003, S. 758-778.

Schweinitz, Jörg/Tröhler, Margrit: »Editorial zum Themenschwerpunkt Figur und Perspektive (2)«, in: montage/av 16/1 (2007), S. 3-11.

Scruton, Roger: The Aesthetics of Music, Oxford: Clarendon Press 1997.

Ders.: Art and Imagination. A Study in the Philosophy of Mind, London: Methuen 1974.

Slaby, Jan: »Möglichkeitsraum und Möglichkeitssinn. Bausteine einer phänomenologischen Gefühlstheorie«, in: Kerstin Andermann/Undine Eberlein, Gefühle als Atmosphären. Neue Phänomenologie und philosophische Emotionstheorie, Berlin: Akademie Verlag 2011, S. 125-138.

Sobchack, Vivian: »What my Fingers Knew: The Cinesthetic Subject, or Vision in the Flesh«, in: Dies., Carnal Thought. Embodiment and Moving Image 
Culture, Berkeley/Los Angeles/London: University of California Press 2004, S. 53-84.

Dies.: The Address of the Eye. A Phenomenology of Film Experience, Princeton: Princeton University Press 1992.

Stein, Edith: Zum Problem der Einfühlung, Freiburg im Breisgau: Herder 2008.

Stueber, Karsten R.: Rediscovering Empathy, Agency, Folk Psychology, and the Human Sciences, Cambridge: MIT Press 2006.

Tan, Ed: Emotion and the Structure of Narrative Film: Film as an Emotion Machine, Mahwah, NJ: Erlbaum 1996.

Vaage, Margrethe Bruun: »Fiction Film and the Varieties of Empathic Engagement«, in: Midwest Studies in Philosophy XXXIV (2010), S. 158-179.

Vendrell Ferran, Ingrid: »Das Wissen der Literatur und die epistemische Kraft der Imagination «, in: Christoph Demmerling/Ingrid Vendrell Ferran: Wahrheit, Wissen und Erkenntnis in der Literatur. Philosophische Beiträge, Berlin: De Gruyter 2014, S. 119-140.

Voss, Christiane: »Einfühlung als epistemische und ästhetische Kategorie bei Hume und Lipps«, in: Robin Curtis/Gertrud Koch (Hg.), Einfühlung. Zu Geschichte und Gegenwart eines ästhetischen Konzepts, Paderborn/München: Fink 2009, S. 31-47.

Voss, Christiane: Der Leihkörper. Erkenntnis und Ästhetik der Illusion, Paderborn: Fink 2013.

Wellbery, David: Stimmung (=Ästhetische Grundbegriffe, Band 5), Stuttgart/Weimar: Metzler 2003, S. 703-33.

Zahavi, Dan: Self and Other, Exploring Subjectivity, Empathy, and Shame, Oxford: Oxford University Press 2014.

Ders.: »Empathy and Direct Social Perception. A Phenomenological Proposal«, in: Review of Philosophy and Psychology 2 (2011), S. 541-558.

Ders.: »Empathy, Embodiment and Interpersonal Understanding: From Lipps to Schutz«, in: Inquiry 53, 3 (2010), S. 285-306.

Zahavi, Dan/Overgaard, Søren: »Empathy without Isomorphism: A Phenomenological Account «, in: Jean Decety (Hg.), Empathy: From Bench to Bedside, Cambridge, MA: MIT Press 2012, S. 3-20. 